\title{
Chameleon-inspired multifunctional plasmonic nanoplatforms for biosensing applications
}

\author{
Yasamin Ziai ${ }^{1}$, Francesca Petronella ${ }^{2}$, Chiara Rinoldi ${ }^{1}$, Paweł Nakielski ${ }^{1}$, Anna Zakrzewska ${ }^{1}$, Tomasz A. Kowalewski ${ }^{1}$,

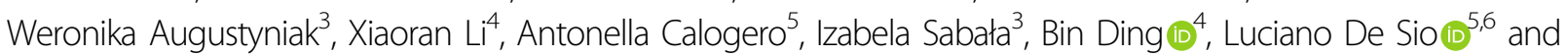 \\ Filippo Pierini (10
}

\begin{abstract}
One of the most fascinating areas in the field of smart biopolymers is biomolecule sensing. Accordingly, multifunctional biomimetic, biocompatible, and stimuli-responsive materials based on hydrogels have attracted much interest. Within this framework, the design of nanostructured materials that do not require any external energy source is beneficial for developing a platform for sensing glucose in body fluids. In this article, we report the realization and application of an innovative platform consisting of two outer layers of a nanocomposite plasmonic hydrogel plus one inner layer of electrospun mat fabricated by electrospinning, where the outer layers exploit photoinitiated free radical polymerization, obtaining a compact and stable device. Inspired by the exceptional features of chameleon skin, plasmonic silver nanocubes are embedded into a poly(N-isopropylacrylamide)-based hydrogel network to obtain enhanced thermoresponsive and antibacterial properties. The introduction of an electrospun mat creates a compatible environment for the homogeneous hydrogel coating while imparting excellent mechanical and structural properties to the final system. Chemical, morphological, and optical characterizations were performed to investigate the structure of the layers and the multifunctional platform. The synergetic effect of the nanostructured system's photothermal responsivity and antibacterial properties was evaluated. The sensing features associated with the optical properties of silver nanocubes revealed that the proposed multifunctional system is a promising candidate for glucose-sensing applications.
\end{abstract}

\section{Introduction}

Currently, bioinspired and biomimetic materials, methods, and algorithms are widely used, as nature is an excellent source of inspiration for a wide range of procedures and designs. There has been a comprehensive classification of inspiration and mimetics from nature, including processes, designs, and materials ${ }^{1}$. One of the critical aspects of bioinspired materials lies in their ability to modify, recover and rebuild their structure under environmental changes over time ${ }^{2}$. Bioinspiration is useful

Correspondence: Luciano De Sio (luciano.desio@uniroma1.it) or Filippo Pierini (fpierini@ippt.pan.pl)

${ }^{1}$ Department of Biosystems and Soft Matter, Institute of Fundamental Technological Research, Polish Academy of Sciences, Warsaw 02-106, Poland ${ }^{2}$ Institute of Crystallography CNR-IC, National Research Council of Italy, Via Salaria Km 29,300, 00015 Monterotondo - Rome, Italy

Full list of author information is available at the end of the article in various applications such as architecture ${ }^{3}$, robotics ${ }^{4}$, textiles ${ }^{5}$, and, more specifically, biomaterials used for energy storage $^{6}$, sensors ${ }^{7}$, water filtration ${ }^{8}$, wearable electronics ${ }^{9}$, and biomedical applications ${ }^{10}$.

Many living organisms have developed the ability to change their properties via physical interaction of external visible light with their nanostructured natural structures. Coloration is an excellent example of these changes, which can be described as "structural." Indeed, this phenomenon is generated by periodic arrays of nano- and microstructures, known as photonic crystals (PCs), which create colors through optical interference effects ${ }^{11}$. Animals such as chameleons are capable of locomotion for consumption and escape, using coloration for attraction, warning, and disguise ${ }^{12}$. In addition, chameleons cannot generate any body heat, but the color of their skin can be

\section{(c) The Author(s) 2022}

(c) (i) Open Access This article is licensed under a Creative Commons Attribution 4.0 International License, which permits use, sharing, adaptation, distribution and reproduction c. in any medium or format, as long as you give appropriate credit to the original author(s) and the source, provide a link to the Creative Commons license, and indicate if changes were made. The images or other third party material in this article are included in the article's Creative Commons license, unless indicated otherwise in a credit line to the material. If material is not included in the article's Creative Commons license and your intended use is not permitted by statutory regulation or exceeds the permitted use, you will need to obtain permission directly from the copyright holder. To view a copy of this license, visit http://creativecommons.org/licenses/by/4.0/. 
used as feedback to regulate their body temperature ${ }^{13,14}$. In particular, the skin of the chameleon is characterized by two superimposed layers of iridophores, namely, cells responsible for skin coloration. The lower layer provides thermoregulation, while the upper layer controls the color changes. Iridophores of the upper layer contain guanine nanocrystals in a regular packed lattice. These guanine nanocrystals are interposed to the cytoplasm, resulting in an arrangement where a higher refractive index material (guanine) is assembled with a lower refractive index material (cytoplasm), thus generating a lattice-like structure similar to that of photonic crystals ${ }^{15}$. This lightmatter mechanism can inspire the design of biosensors to produce photo/photothermal-driven devices that can monitor changes without requiring any external energy input.

Biosensors are defined as devices that involve specific biochemical and physical reactions mediated by immune systems, tissues, organelles, or biomimetic components to detect chemical compounds, usually by electrical, thermal, or optical signals ${ }^{16}$. In recent years, the development of nanomaterials for the ultrasensitive detection of biological species has received significant attention because of their unique optical, electronic, chemical, and mechanical properties. Different nanomaterials have been investigated to determine their properties and possible biosensing applications ${ }^{17}$. Considering the increasing prevalence of diabetes and hypoglycemia, glucose sensing has received much interest in recent years ${ }^{18,19}$. Recently, there have been outstanding investigations into electrochemical ${ }^{20,21}$ and spectroscopic ${ }^{22}$ methods for glucose monitoring. The main challenge, which concerns fast, personalized tracking, consists of sensing the glucose rate without bloodletting, thus making it as user-friendly as possible. Glucose levels can be measured using urine ${ }^{23}$, perspiration $^{24}$, saliva ${ }^{25}$, ocular fluid ${ }^{26}$, and even breath analysis ${ }^{27}$. Within this framework, over the past several decades, soft elastic materials have become of great interest due to their body compliance and are being used for wearable electronics and wearable biosensors. Compared to physical sensors, these devices require the integration of specific bioreceptors into sensing elements that are capable of recognizing target analytes in complex samples at physiologically significant concentrations. To enable ultrafast glucose monitoring, it is generally necessary to entrap the enzyme glucose oxidase within a flexible device. Elsherif et al. fabricated wearable contact lens optical sensors based on hydrogel networks for continuous glucose measurement under physiological conditions ${ }^{28}$. Organic biosensors can be processed inexpensively on flexible substrates by using printing techniques; the organic material can also be tailored to adjust the properties ${ }^{17}$. In addition, other properties such as wearability, flexibility, and the possibility of fabricating these platforms using low-cost processes ensure favorable prospects for these materials $^{29,30}$.

Among materials, hydrogels are considered one of the best alternatives for the fabrication of organic biosensors. Hydrogels are defined as crosslinked polymeric networks with an extensive water swelling capacity and can be synthesized by a simple reaction of one or more monomers. The essential property of these polymeric materials is their ability to swell and retain a significant fraction of water within their structure without dissolving in water ${ }^{31}$. The ability of hydrogels to absorb water arises from the hydrophilic functional groups attached to the polymeric backbone, while their intrinsic resistance to dissolution comes from crosslinks between network chains. Usually, synthetic polymers have well-defined structures that can be modified to yield on-demand degradability and functionality $^{32}$. Hydrogels are engineered to exhibit a significant volume variation in response to small changes in their environmental factors, such as $\mathrm{pH}$, ionic strength, temperature, solvent, and applied electric/magnetic field, which can be used for sensing applications. Recent advancements in polymer science have led to the development of biopolymers suitable for synthesizing various hydrogels. Hydrogels can be responsive to external stimuli such as temperature, $\mathrm{pH}$, or the ionic strength of the surrounding medium ${ }^{33}$. These systems are called smart hydrogels, as they respond immediately to environmental changes. They can be categorized into two classes based on the chemical or physical nature of the crosslinked interactions. Crosslinking due to chemical interactions leads to a permanent junction in the polymer network, while physical interactions are due to either polymer chain entanglements or physical interactions such as ionic interactions, hydrogen bonding, or hydrophobic interactions $^{34,35}$.

Most of the thermoresponsive polymers used in biomedical applications form colloidal systems when the temperature increases, a property known as lower critical solution temperature (LCST) behavior. This process is reversible: polymers return to a sol form when the temperature is lowered below the $\mathrm{LCST}^{27}$. When the macromolecular chains of these thermoresponsive polymers are crosslinked to form hydrogels, the behavior of the final materials is significantly influenced by the volume phase transition temperature (VPTT). Indeed, when the temperature exceeds the typical VPTT, the polymer network shrinks, and the water contained in the hydrogel is expelled from the structure ${ }^{36}$. Thus, crosslinked thermoresponsive hydrogels reversibly switch from a hydrophilic, swollen state to a hydrophobic, shrunken state when heated above the volume phase transition temperature ${ }^{37}$.

Several types of hydrogels based on natural polymers have been investigated in the field of thermoresponsivity ${ }^{38}$; in particular, poly( $\mathrm{N}$-isopropylacrylamide) 
(PNIPAAm) has attracted high interest in recent years. Indeed, PNIPAAm is nonbiodegradable and has a sharp phase transition, with a VPTT close to the human body temperature (at $\sim 32{ }^{\circ} \mathrm{C}$ in pure water) ${ }^{39}$. According to its thermoresponsive behavior, the polymer shape is an extended coil conformation below the VPTT $\left(32^{\circ} \mathrm{C}\right)$, showing a well-defined structure and properties ${ }^{40}$. Interestingly, the VPTT can be finely tuned according to the specific application $^{41}$.

Plasmonic nanoparticles (NPs) are metallic subunits with a dimensional range smaller than the wavelength of visible light; they possess unique physical and optical properties such as localized surface plasmon resonance (LSPR), which is a coherent and dipolar oscillation of the bulk-free electrons localized at the metallic/dielectric interface. Moreover, the confinement effect confers reactivity and mechanical, electromagnetic, chemical, and optical properties that differ from those of bulk metals ${ }^{42,43}$. The SPR band is much stronger for plasmonic NPs than for other metals, as their SPR band appears in the visible region of the electromagnetic spectrum. In contrast, other metals have weak bands in the ultraviolet (UV) region. Among all metallic NPs, gold $(\mathrm{Au})$ and silver (Ag) NPs have the most attractive physical properties for biosensing-related applications. Because of their excellent chemical stability and biocompatibility, gold nanoparticles (AuNPs) are the most investigated in this research area, most frequently for drug delivery systems and cancer therapy applications ${ }^{44}$. Due to their excellent chemical stability and biocompatibility ${ }^{45}$, AuNPs are widely used in drug delivery systems and cancer therapy. On the other hand, silver nanoparticles (AgNPs) offer better results in terms of sensitivity ${ }^{46}$, and thus, they are mainly used for sensing applications. AgNPs with different shapes, including cubes, wires, spheres, triangles, and rods, have distinct crystal faces. In particular, the contact area in silver nanocubes (AgNCs) is more significant than that in nanospheres or other morphologies, resulting in more grain boundaries. The higher the percentage of contact area on a specific NP shape, the more active the NP will be $^{47,48}$. Accordingly, a few studies have recently reported the combination of thermoresponsive polymer composites and metal NPs to enhance their specific properties.

He et al. used NIPAAm and sodium acrylate as functional monomers to form a hydrogel layer, and AgNPs were loaded inside them for antibacterial purposes. It was possible to effectively kill the contacting and surrounding bacteria. The attached dead bacteria were then detached as a result of the increased surface hydrophilicity and degree of surface swelling by reducing the temperature below the $\mathrm{VPTT}^{49}$. AgNPs are frequently used as antibacterial agents to effectively reduce bacterial viability. Bactericidal surfaces can prevent the formation of biofilms for a certain period until AgNPs are completely consumed. This behavior shows that the antibacterial property can be maintained only for a limited time, motivating researchers to design and construct environment-responsive antibacterial surfaces, including thermoresponsive, pH-responsive, enzyme-responsive, and photoresponsive surfaces ${ }^{49}$.

Ultrathin fibers fabricated by electrospinning have attracted much attention for various biomedical applications, such as tissue engineering ${ }^{50}$, drug delivery ${ }^{51}$, wound dressing, bioscaffolds ${ }^{52}$, and biosensors ${ }^{53,54}$. The nanofibers obtained using this technique can have a size range close to $100 \mathrm{~nm}$, extremely high surface-to-volume ratio, tunable porosity, and controllable properties and functionalities ${ }^{55,56}$.

In this article, taking inspiration from light-matter interactions and the functionalities of chameleon skin, we report the development of a fast, sensitive, smart, antibacterial glucose-sensing system. The proposed innovative system consists of hydrogels as three-dimensional networks responding to stimuli and electrospun fibers with their unique structure and properties. To fabricate such smart platforms, a PNIPAAm-derivative plasmonic hydrogel containing AgNCs was synthesized. Using the electrospinning technique, a mat consisting of polycaprolactone (PCL) and polyethylene oxide (PEO) was also prepared, providing ultrathin fibers with a high surface area in a facile and inexpensive way. The composites consisted of two outer layers of hydrogel and an inner layer of electrospun mat. Their resulting unique hierarchical structure was deeply characterized, while structural and optical properties were thoroughly investigated to embrace the full potential of the platform. In addition, the antibacterial and photothermal responses of the samples were evaluated. Finally, the application of the developed nanostructured platform as an LSPR biosensor was evaluated by quantifying the glucose level in the human body. The change in glucose concentration in human urine produced a shift in the LSPR bands of AgNCs. By applying the proposed organic-based inexpensive, smart, nanostructured platform, glucose levels in urine can be rapidly and easily monitored without any external energy input requirement, equipment, or bloodletting.

\section{Materials and methods \\ Materials}

N,N-isopropylacrylamide (NIPAAm, 97\%, Sigma Aldrich, Poland), N-isopropylomethacrylamide (NIPMAAm, 97\%, Sigma Aldrich, Poland), N,N'-methylene bisacrylamide (BIS-AAm, 99.5\%, Sigma Aldrich, Poland), 2-hydroxy-4'-(2-hydroxyethoxy)-2-methylpropiophenone (Irgacure 2959, 98\%, Sigma Aldrich, Poland), silver nanocubes (AgNCs, $75 \mathrm{~nm}$, PVP, Nanocomposix, USA), polycaprolactone (PCL, Mn $80 \mathrm{kDa}$ ), polyethylene oxide 
(PEO, Mn $100 \mathrm{kDa}$ ), dichloromethane (DCM, POCh, Poland), and N,N-dimethylformamide (DMF, POCh, Poland) were purchased and used as received.

\section{Hydrogel precursor sol including silver nanocubes}

To prepare the hydrogel precursor solution at a concentration of $4.8 \mathrm{wt} \%, 578.13 \mathrm{mg}$ of NIPAAm, $15.63 \mathrm{mg}$ of NIPMAAm, and $31.25 \mathrm{mg}$ of BIS-AAm were added to $12.5 \mathrm{mg}$ of Irgacure 2959. This mixture was dissolved in $10 \mathrm{ml}$ of deionized water to reach a $95.2 \mathrm{wt} \%$ solution. The mixture was wrapped in aluminum foil for protection from light and stirred overnight until complete dissolution.

To optimize the final embedding of AgNCs into the solution, a suspension of AgNCs with three different proportions $-2 \%, 5 \%$, and $20 \% \mathrm{wt} / \mathrm{wt}_{\text {monomer }}$-was added to the hydrogel precursor solution.

\section{Preparation of electrospun fibers}

First, 12\% (w/v) PCL/PEO, which was prepared at the ratio of 75:25 (w/w) PCL/PEO, was dissolved into 80:20 $(\mathrm{v} / \mathrm{v})$ dichloromethane:dimethylformamide (DCM:DMF) solution and stirred overnight at room temperature by using a magnetic stirrer prior to electrospinning. The desired structure was achieved by performing the procedure with a flow rate of $300 \mu \mathrm{L} \mathrm{h}^{-1}$ and a positive voltage of $15 \mathrm{kV}$. The rotating drum used to collect fibers was placed $13 \mathrm{~cm}$ away from the $21 \mathrm{G}$ needle tip and rotated at $500 \mathrm{rpm}$. The electrospinning process was performed at a temperature of $\sim 21-22{ }^{\circ} \mathrm{C}$ with $50 \%$ humidity. Electrospun fibers were placed on glass slides, and small circular holes were cut into the fibrous mat with a laser cutting system (Optec) on a high-precision motorized X-Y-Z linear stage (Newport, Irvine, CA) controlled by a PC. A picosecond laser with a wavelength of $355 \mathrm{~nm}$, a $200 \mathrm{kHz}$ repetition rate of the laser pulse, and a minimum laser spot size of $3 \mathrm{~mm}$ was used. The laser power was $\sim 0.5 \mathrm{~W}$ (7\%), the translation speed of the laser head was set at $2000 \mathrm{~mm} \mathrm{~s}^{-1}$, and the laser head moved eleven times along the same line to cut the microstructures correctly. A $\times 5$ objective (Mitutoyo, Japan) was used to focus laser pulses. The hole is necessary to enable the laser beam to pass through just the hydrogel layer and, in return, to avoid any possible interference generated by the electrospun membrane during glucose quantification.

\section{Fabrication of the nanostructured platform}

To fabricate the final platform structure, a tailordesigned metal mold was used as a template. A $2.5 \mathrm{ml}$ hydrogel precursor solution was poured into the mold; then, an electrospun fiber mat was cut into strips $(6 \times$ $1 \mathrm{~cm}$ ) and placed gently on the solution. Next, $2.5 \mathrm{ml}$ of hydrogel was poured over the fibrous strips to obtain the final platform. The obtained system was placed in a petri dish inside an ice bath and irradiated with a UV lamp for $2 \mathrm{~min}$. Samples were dried in a hood overnight and rinsed with deionized water for one week to remove all the unpolymerized material and the residual photoinitiator.

\section{Structural characterization}

To ensure the presence of AgNCs and their distribution in the hydrogel matrix, each separate layer and the whole final construct were characterized morphologically.

Field emission scanning electron microscopy (FE-SEM) and scanning electron microscopy (SEM) were performed with FEI Nova NanoSEM 450 and JEOL JSM-6390LV microscopes, respectively. Hydrogel disks and the final construct were frozen in liquid nitrogen before crosssectional cutting, and then, freeze-dried samples were obtained. Before imaging, samples were sputtered with gold layers of approximately $8 \mathrm{~nm}$ in thickness using a SC7620 Polaron mini sputter coater (Quorum Technologies Ltd., Ashford, UK). The samples were freeze-dried, and then, the lyophilized materials were cut into small pieces, immersed in ethanol, and placed on a copper mesh with an amorphous carbon membrane. For transmission electron microscopy (TEM), samples were analyzed on an FEI Talos F200X TEM (acceleration voltage of $200 \mathrm{kV}$ ) after four hours of drying in a vacuum drier.

Energy-dispersive X-ray spectroscopy (EDX) was performed in STEM mode. For this purpose, a high-angle annular dark-field detector and a Super-X EDX system with four silicon drift detectors (SDDs) were used. The sample surface topography was evaluated by atomic force microscopy (AFM, Ntegra, NT-MDT) equipped with a silicon cantilever (NSG01, NT-MDT, tip radius $10 \mathrm{~nm}$ ). Measurements were performed in semicontact mode, with a resonance frequency of $150 \mathrm{kHz}$ and $500 \times 500$ points per image. The hydrodynamic diameter of AgNCs was analyzed by dynamic light scattering (DLS) with a Zetasizer Nano ZS system (Malvern, UK). Dispersions of $0.1 \mathrm{wt} \%$ AgNCs in water were prepared, and samples were loaded into quartz microcuvettes and measured in triplicate, with the mean value recorded.

\section{Chemical characterization}

A Multiskan GO spectrophotometer (Thermo Scientific, USA) was used to collect data for ultraviolet-visible (UV-Vis) spectra. The UV-Vis spectrophotometer scanned from $400 \mathrm{~nm}$ to $1000 \mathrm{~nm}$ at $5 \mathrm{~nm}$ intervals. X-ray scattering (XRD) analyses were performed to confirm the presence of AgNCs in the hydrogel matrix and to detect any potential changes in the hydrogel structure caused by the inclusion of AgNCs. Measurements were carried out on a Bruker D8 Discover diffractometer in reflection mode using Bragg-Brentano geometry. The analysis was conducted in the angular range (2theta) of $5-50^{\circ}$. Data were collected with a step of $0.02^{\circ}$ per $1.0 \mathrm{~s}$ at each point. 
Fourier transform infrared (FT-IR) spectroscopy was used to characterize the functional groups in each layer. FT-IR analyses were conducted in attenuated total reflectance (ATR) mode with a Bruker Vertex70 FT-IR spectrometer and carried out in the wavenumber range of $400-4000 \mathrm{~cm}^{-1}$ with a resolution of $2 \mathrm{~cm}^{-1}$ and 12 scans for each sample.

\section{Mechanical testing}

Macrotensile measurements were performed using a tensile tester (Model EZ 50, Lloyd Instruments strength machine, Germany). Specimens were obtained by cutting the final construct into strips of a size of $6.0 \times 1.0 \mathrm{~cm}^{2}$. All samples were mounted between holders at a distance of $1 \mathrm{~cm}$ (Pneumatic Action grips, Elancourt, France). Tensile testing was conducted at a rate of $5 \mathrm{~mm} \mathrm{~min}^{-1}$ at room temperature $\left(21^{\circ} \mathrm{C}\right)$. Specimens were kept wet by immersing them in water at the test temperature. A $50 \mathrm{~N}$ load was set on the instrument.

\section{Antibacterial characterization}

The antibacterial activity of the hydrogel disks was tested on the Staphylococcus aureus 8325-4 strain. One milliliter of overnight inoculum was added to $50 \mathrm{ml}$ of liquid tryptic soy broth (TSB) and incubated at $37^{\circ} \mathrm{C}$ with shaking $(80 \mathrm{rpm})$ until the cultures reached an $\mathrm{OD}_{600}$ of 0.6. The bacterial cells were harvested by centrifugation, and the palette was resuspended in phosphate-buffered saline (PBS) until reaching an $\mathrm{OD}_{600}$ of 1.0 corresponding to $10^{8} \mathrm{CFU} \mathrm{ml}{ }^{-1}$, followed by further dilution to obtain the desired number of bacteria in $50 \mu \mathrm{L}$, namely, 1000 , $10,000,100,000$, and 1,000,000.

Fifty microliters of bacteria were placed on hydrogel disks and spread gently and uniformly over the whole surface. Control samples were made by pipetting $50 \mu \mathrm{L}$ of bacterial suspensions into Eppendorf tubes. These samples were then incubated at room temperature $\left(\sim 20^{\circ} \mathrm{C}\right)$ for $4 \mathrm{~h}$. Afterward, solutions were taken from the hydrogel disks and subjected to serial dilutions to perform a droplet test: $5 \mu \mathrm{L}$ of each dilution of every sample was placed on TSB agar plates and incubated at $37^{\circ} \mathrm{C}$ overnight, and then, the colonies were counted to estimate the number of bacteria in each sample.

\section{Photothermal responsivity characterization}

The thermo-optical setup used a TEM00 mode CW diode-pumped solid-state laser (Laser Quantum) operating at $532 \mathrm{~nm}$ in the high absorption range of AgNCs (longitudinal plasmon band). A high-resolution thermal camera (FLIR, A655sc) was used to map and identify both the spatial heating distribution and the temperature profile under laser illumination. The camera produced thermal images of $640 \times 480$ pixels with an accuracy of $\pm 0.2^{\circ} \mathrm{C}$; it worked seamlessly with a proprietary software program (FLIR ResearchIR Max) to record and process the thermal data acquired by the camera.

\section{Glucose sensing tests}

The absorption spectra of the P(NIPAAm-co-NIPMAAm) hydrogel samples embedded with AgNCs were collected by using a UV-Vis spectrometer (USB 2000, Ocean Optics). The sensitivity of the AgNCs was tested by preparing four standard solutions of D-glucose in water at the same concentrations used to build the calibration curve for the hydrogel samples. A volume of AgNCs (from a colloidal dispersion of $1 \mathrm{mg} / \mathrm{mL}$ ) was added to each glucose solution suitable to achieve the desired AgNC: water mass ratio. The resulting dispersions were kept under magnetic stirring for $5 \mathrm{~min}$, and then, the corresponding absorption spectra were collected.

Hydrogel samples were placed on a glass substrate, with $50 \mu \mathrm{m}$ glass microspheres added as spacers, and then covered with a glass slide $(100 \mu \mathrm{m}$ thick). The resulting gap was infiltrated via capillary flow, with $50 \mu \mathrm{L}$ of the solution being studied. Samples were allowed to rest for $10 \mathrm{~min}$ to promote a uniform and complete distribution of the solution within the samples before evaluating the optical response as a function of local refractive index changes. Each measurement was performed by examining five different areas of the hydrogel samples. The spectral shifts $(\Delta \lambda)$ were reported as the mean value \pm standard deviation of five different measurements.

To construct the D-glucose calibration curve, hydrogel samples embedded with AgNCs were infiltrated with $50 \mu \mathrm{L}$ of standard water solutions of D-glucose at concentrations from $2 \mathrm{mM}$ to $8 \mathrm{mM}$, and the respective absorption spectra were collected to measure the wavelength of the respective plasmonic peak $\left(\lambda_{\text {glu }}\right)$. To quantify the $\Delta \lambda$ value associated with the respective $\mathrm{D}$-glucose standard solution, the wavelength of the plasmonic peak from the absorption spectrum of a P(NIPAAm-co-NIPMAAm)/AgNC sample was infiltrated with Milli-Q water $\left(\lambda_{\text {water }}\right)$ and used as a reference. $\Delta \lambda$ was then calculated as the difference between $\lambda_{\text {water }}$ and $\lambda_{\text {glu }}$, and the calibration curve was obtained by plotting $\Delta \lambda$ as a function of the concentration of standard solutions of D-glucose.

The limit of detection (LOD) was calculated as 3 times the standard deviation of the intercept, calculated from the linear interpolation resulting from plotting the $\Delta \lambda$ values as a function of the concentration of standard solutions ${ }^{57}$.

The D-glucose concentration in human urine samples was calculated by following the standard addition method. A defined volume $(0 \mathrm{~mL}, 0.2 \mathrm{~mL}, 0.4 \mathrm{~mL} 0.8 \mathrm{~mL}$, and $1 \mathrm{~mL}$ ) of a $10 \mathrm{mM}$ D-glucose standard solution was introduced into $2 \mathrm{~mL}$ volumetric flasks previously filled with $1 \mathrm{~mL}$ of a human urine sample. The resulting solutions were suitably diluted with Milli-Q water and mixed. 
Subsequently the solutions were used to infiltrate $\mathrm{P}$ (NIPAAm-co-NIPMAAm)/AgNC samples to acquire the corresponding absorption spectra. The resulting $\Delta \lambda$ values were plotted as a function of the concentration of standard solutions, thus obtaining the standard addition plot. To obtain statistically reliable data, five different solutions were prepared, infiltrated into the hydrogel, and analyzed for each condition studied.

\section{Results and discussion}

\section{Bioinspired nanostructured platform}

Chameleon skin, shown in Fig. 1a, offered inspiration for the multilayer structure described in this article. The rapid active tuning of skin hue has been described in only a handful of species and generally involves structural rather than pigmentary components. Multilayer nanoreflectors cause these structural changes by alternating high and low refractive indices that generate the interference of light waves. In a similar way, the unique characteristics of the structure of chameleon skin are primarily due to the nano- and microstructured arrangements of chameleon skin tissue, giving rise to photonic crystals (PCs). The layer underneath revealed the presence of fibroblast cells, collagenous fibrils, nerve axons, melanosomes, and mast cells in the connective tissue core. This layer has a general structure similar to that of the electrospun fibrous mat. The structure of the particles in chameleon skin and their similarity to plasmonic nanocubes (Fig. 1b), resulting in the light-matter interaction of the skin and the resemblance between the particle fibrous tissue layers, were the most significant features imitated in the fabrication of this multilayer structure.

The scheme illustrated in Fig. 1c shows the structure of the final construct. An electrospun fiber mat obtained from a blend of poly( $\varepsilon$-caprolactone)/polyethylene glycol (PCL/PEO) was placed between two layers of nanostructured hydrogel based on $\mathrm{N}$-isopropylacrylamide-co$\mathrm{N}$-isopropylmethacrylamide $\mathrm{P}$ (NIPAAm-co-NIPMAAm) with embedded AgNCs. The structure of the guanine particles existing in the chameleon skin (Fig. 1a, right panel) was replicated in the proposed plasmonic nanoplatform by introducing silver nanocubes (Fig. 1b). Therefore, the development of a structure evoking guanine nanocrystals in the cytoplasm of chameleon iridophores and the resemblance between the particle fibrous tissue layer were the most important features when fabricating this multilayer structure. This system is designed to show photothermal responsivity due to the nanostructure of the particles and the nature of the P(NIPAAmco-NIPMAAm) hydrogel. The electrospun mat between

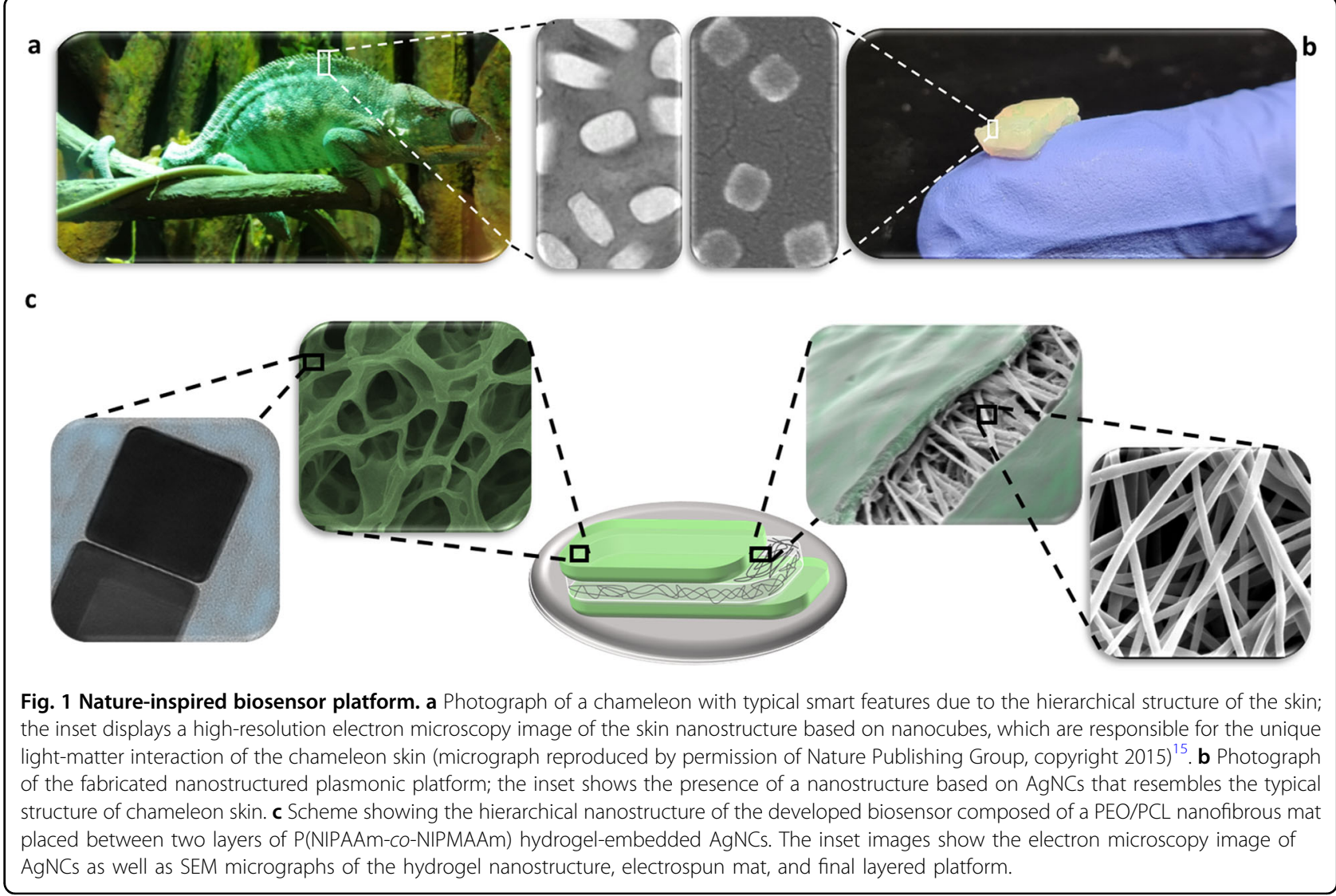




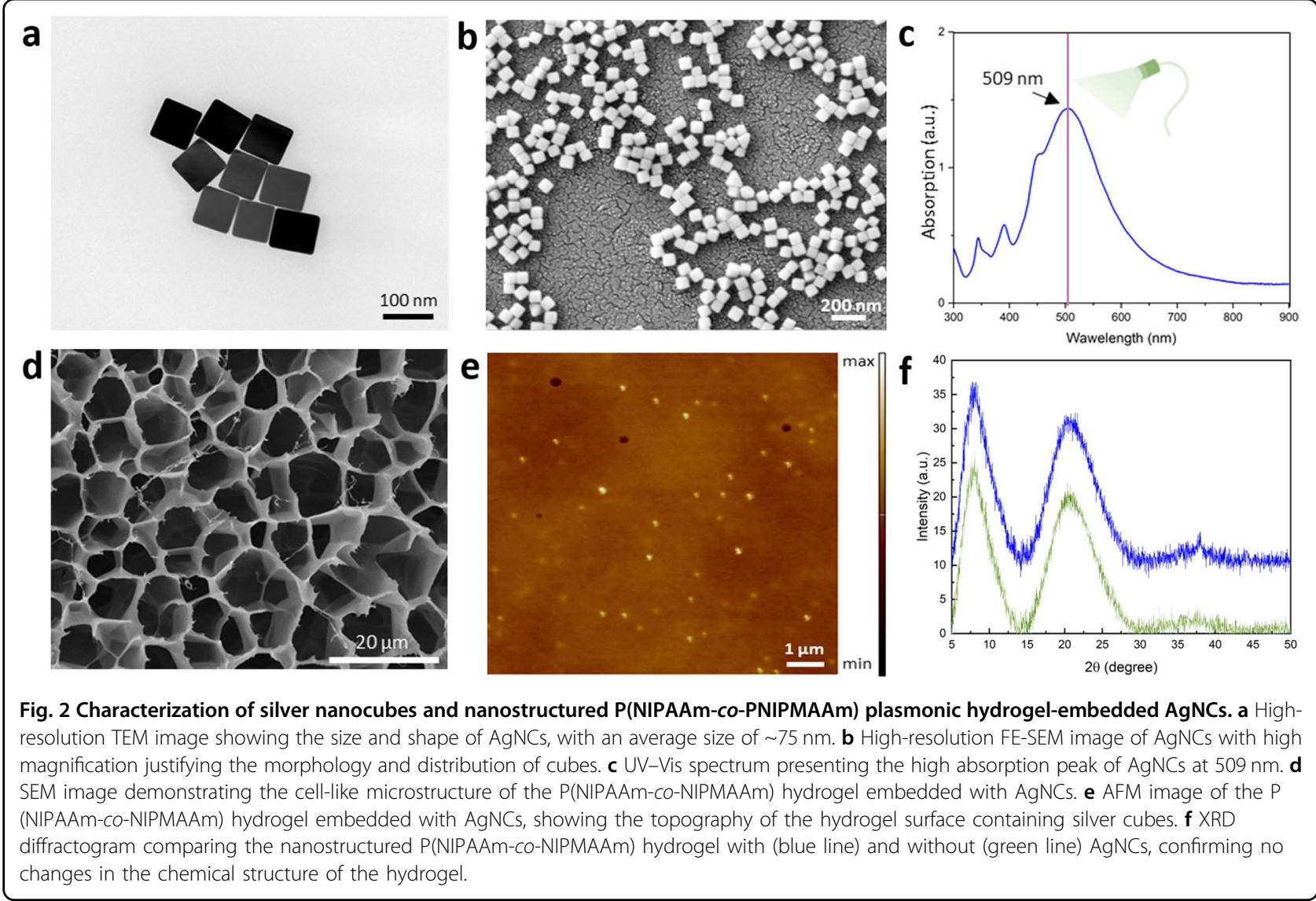

hydrogel layers provides mechanical support and makes it easier to handle as a biomedical device ${ }^{58}$.

\section{Silver nanocubes embedded in the thermoresponsive $P$ (NIPAAm-Co-NIPMAAm) hydrogel}

AgNCs introduced into the proposed platform have an average dimension of $75 \pm 3 \mathrm{~nm}$, as shown by both FESEM and TEM images. TEM and EDS elemental mapping images are shown in Fig. 2a and Fig. S1, indicating the size, shape, and distribution of the AgNCs. Field-emission SEM images of the cubes are also presented in Fig. S2 and Fig. 2b, with two different levels of magnification. AgNCs have a perfect cubic shape: the edges and corners are perfectly visible, revealing that the synthesis and the utilized protocol produced the desired shape. This observation is good evidence of the homogeneity and regularity of the cubes that we used in this study. It is worth pointing out that even during the sample preparation for imaging, where the medium of the sols was completely removed, there was no sign of visible aggregations in the microscopic images, and the AgNCs were well distributed. Thus, the stability and homogeneity of the precursor solution used to prepare the hydrogel were proved. The DLS results (Fig. S3) showed a single peak: an average hydrodynamic diameter of AgNCs of approximately
$109 \mathrm{~nm}$. The data appear consistent since one single population is present; thus, one single peak in the DLS graph is expected. The visible difference between the DLS results and the reported morphological determination of the size of the cubes obtained by electron microscopy is because DLS is a technique developed to analyze nanoobjects and, as a result, determines the equivalent hydrodynamic size of the particles. It is worth noting that the hydrodynamic radius of a particle is generally more prominent than its physical radius. Moreover, DLS detects the radius of an equivalent spherical particle, having the same rheological behavior like that of our single nanocubes dispersed in the analyzed colloidal sol. Therefore, it is not possible to directly compare the diameter obtained by electron microscopy with DLS results. In any case, the DLS outcomes are consistent with the previous quantitative data obtained by FE-SEM and TEM in terms of sol stability and absence of any aggregates under the utilized experimental conditions. As shown in Fig. 2c, the UV-Vis spectra of AgNCs show a strong light absorbance peak centered at $\lambda=509 \mathrm{~nm}$, which is the expected wavelength for AgNCs with the described shape and size to absorb light due to their LSPRe.

In recent years, PNIPAAm has been extensively studied, as it is a thermoresponsive hydrogel that undergoes 
reversible phase transitions at specific temperatures. The coil-to-globule transition of the polymer consists of at least two different thermal processes. First, water molecules around the hydrophobic isopropyl group and hydrophilic amide groups can rearrange in bulk water. Second, intra- and interchain hydrogen bonds are created between amide groups, and this phenomenon is accompanied by a loss in polymer chain-water hydrogen bonds. This transition depends on several factors, e.g., polymer concentration, crosslinking agent concentration, and copolymerization with other monomers. To increase the VPTT, two monomers, NIPAAm and NIPMAAm, were copolymerized. The VPTT of PNIPAAm in water is approximately $32^{\circ} \mathrm{C}$, while that of PNIPMAAm is higher, $\sim 42^{\circ} \mathrm{C}$. According to the literature, the VPTT of the resulting copolymer with a smaller quantity of NIPMAAm (as in our case) should increase to $\sim 33-34{ }^{\circ} \mathrm{C}$ when macromolecule chains are not crosslinked ${ }^{59}$. In our case, the use of $N, N^{\prime}$-methylene bisacrylamide (BIS-AAm) shifted the VPTT. This shift led to the formation of a broad peak due to the faster reaction of BIS-AAm than that of NIPAAm and NIPMAAm during polymerization.

The SEM micrograph of the hydrogel reported in Fig. $2 \mathrm{~d}$ was taken from the cross-section of the samples, which were dried before use. The image shows the cell-like network structure of the hydrogel with a pore size ranging from $8 \mu \mathrm{m}$ to $10 \mu \mathrm{m}$. The cell-like structure with interconnected pores is highly significant, as it makes it possible for the network to absorb a great amount of water and gain the desired hydrogel properties ${ }^{60}$. An SEM image of the neat hydrogel network not filled with AgNCs is shown in Fig. S4. The cell-like structure shows that there are no visible differences in the structure and pore sizes with and without the introduction of AgNCs. It can be clearly seen, however, that the presence of AgNCs in the hydrogel network can impart the hydrogel with new optical properties due to the optical properties of welldispersed AgNCs (Fig. S5a, b). As shown in the AFM topography of the hydrogel with embedded AgNCs (Fig. $2 \mathrm{e})$, there were few signs of AgNCs on the hydrogel surface. Therefore, it may be concluded that AgNCs (20 wt\%) are trapped within the porous network of the hydrogel, which also appears on the surface. This process gives rise to both the photothermal responsivity and antibacterial properties of the material and affects the platform's sensing features for the ultimate application of the system. Two more different concentrations of AgNCs in the hydrogel (i.e., 2 and $5 \mathrm{wt} \%$ ) were tested, and the AFM images in Fig. S6a, b show the distribution of AgNCs on the surface of the hydrogel. However, $20 \mathrm{wt} \%$ AgNCs was selected due to a sufficient number of AgNCs, providing effective antibacterial and photothermal responsiveness, thus allowing a more sensitive sensing capability. The effect of AgNCs on the chemical structure of the hydrogels can also be proven through the comparison between XRD measurements of the pure hydrogel and the hydrogel embedded with AgNCs. Typical broad peaks at $\sim 8.0$ and $20.7^{\circ}$ are shown in the XRD diffractogram (Fig. $2 \mathrm{f}$ ), which is attributed to the polymer's amorphous state. The presence of AgNCs is proved by the small additional peak at $38.0^{\circ}$, but otherwise, there is no other visible change in the XRD diffractogram, which demonstrates that the addition of AgNCs does not change the chemical structure of the hydrogel. Thus, adding nanocubes does not affect the crystallinity of the polymer; hence, no changes in the XRD diffractogram are observed.

\section{Electrospun nanofibers and nanostructural platform fabrication}

A schematic of the final composite fabrication is shown in Fig. 3a. First, the nanofibrous mat was electrospun from the prepared solution and collected on a planar collector. As previously described, the electrospun mat was then subjected to laser cutting, and $3 \mathrm{~mm}$-diameter holes were cut into it to ensure the passage of light through the entire layered platform for biosensing tests. Meanwhile, after argon bubbling to remove oxygen molecules, the hydrogel precursor solution was added to a prepared rectangular mold. The electrospun fiber mat was then added on top of the hydrogel precursor solution, and a layer of the hydrogel precursor solution was poured gently over it. UV-irradiated polymerization was performed while the platform was in an ice bath to control the heat generated during the polymerization process. The crosslinked hydrogel was removed from the mold and cut into strips for further investigations.

Polycaprolactone (PCL) is a biodegradable polyester commonly used in the biomedical field for drug delivery systems and three-dimensional (3D) scaffolds for tissue engineering $^{61}$. This polymer is biocompatible and biodegradable, and owing to its semicrystalline and hydrophobic nature, PCL has a very slow degradation rate and mechanical properties suitable for several applications. The transformation of this polymer to nanofibers to achieve a desirable structure by electrospinning is quite easy, thus revealing its excellent value in terms of final applicability/ applications. PCL is compatible with a wide range of other polymers and is thus a great choice when multifunctional mats are needed. PEO is also a biocompatible and biodegradable polymer with a hydrophilic structure and watersoluble, nonionic, and nontoxic properties; it is introduced here as a component to modify the hydrophilic properties of the PCL mat. The combination of these two polymers increases the hydrophilicity of PCL/PEO nanofibers compared to pure $\mathrm{PCL}^{62}$ and improves the mechanical properties compared to pure $\mathrm{PEO}^{63}$.

As shown in the SEM image of the electrospun fibers in Fig. 3b, PCL/PEO nanofibers with fiber dimensions of 

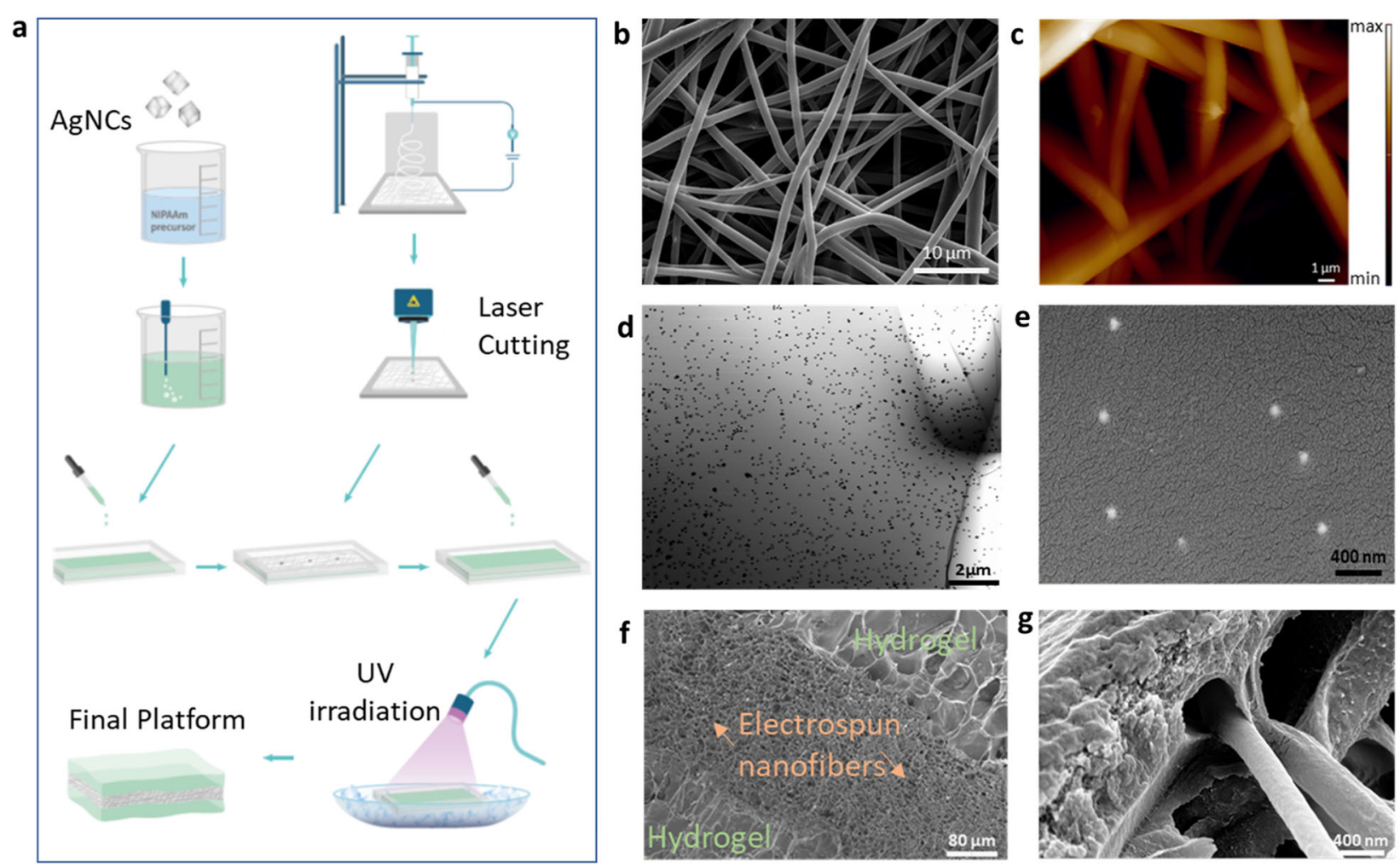

Fig. 3 Characterization of the electrospun nanofibers and nanostructured platform. a Scheme showing the preparation procedure of the nanostructured platform: starting from the preparation of a hydrogel solution, electrospinning of a PEO/PCL nanofibrous mat, followed by assembly of the platform, with free-radical polymerization of the final construct as the final step. b SEM micrograph showing the morphology of spun PEO/PCL nanofibers with a fiber diameter of $1 \mu \mathrm{m}$. c The surface topography of PEO/PCL nanofibers observed with AFM shows a soft fiber surface. The Z-scale bar corresponds to $7 \mu \mathrm{m}$. d The high-resolution TEM image of the plasmonic hydrogel composite in the final platform shows the size and distribution of AgNCS in the hydrogel matrix. e High-resolution FE-SEM top-view image of the platform surface, confirming the presence of AgNCs. $\mathbf{f}$ Crosssectional SEM image of the platform showing the PEO/PCL electrospun mat placed between two layers of P(NIPAAm-Co-NIPMAAm) hydrogel. $\mathbf{g}$ A high-resolution cross-sectional FE-SEM image of the platform, confirming the interpenetration of nanofibers within the hydrogel layers.

approximately $1 \mu \mathrm{m}$ were successfully fabricated. The fibers obtained were uniform, regular, and bead-less, and they were randomly oriented and well distributed throughout the whole mat. They were also defect-free, showing no signs of breaking or deformation. In addition, the surface of these fibers was observed via AFM, as shown in Fig. 3c: the fibers appeared uniform and without defects. Figure $3 \mathrm{~d}$ shows the TEM image of AgNCs in the final platform, confirming the presence of AgNCs in the hydrogel matrix. It can be observed that the nanoparticles are well dispersed throughout the hydrogel network; moreover, there is no sign of nanofiller aggregation. Highresolution TEM and EDS images in Fig. S7a, b show the high-magnification visualization of AgNCs in the hydrogel network. The uniform distribution and nonaggregation of AgNCs are confirmed here at nanometric scales. Fig. S8 shows the AFM topography of the composite, evidencing the presence of AgNCs on the surface of the hydrogel. There were no signs of fibers on the surface topography of the hydrogel composite, thus confirming the homogeneous hydrogel layering on the platform. Figure 3e shows a top-view FE-SEM image of the platform, revealing a plain surface and the presence of AgNCs in the structure. Figure $3 \mathrm{f}$ shows an FE-SEM image of the platform's cross-section, highlighting the platform's layered structure composed of an electrospun fibrous mat placed between two layers of P(NIPAAm-co-NIPMAAm) hydrogel embedded with AgNCs. The nanometric scale interface and interpenetration of fibers in the hydrogel structure are visible in the cross-sectional FE-SEM image presented in Fig. 3g. The interpenetrated fibers in the cross-section support the idea of a compact platform and intimate anchorage between the layers (Fig. $3 \mathrm{~g}$ ), which play a crucial role in the mechanical properties and stability of the system.

\section{Mechanical testing of the fabricated platform}

Hydrogels possess poor mechanical properties due to their network structure and the retainment of significant amounts of water. Acrylate-based hydrogels are no 


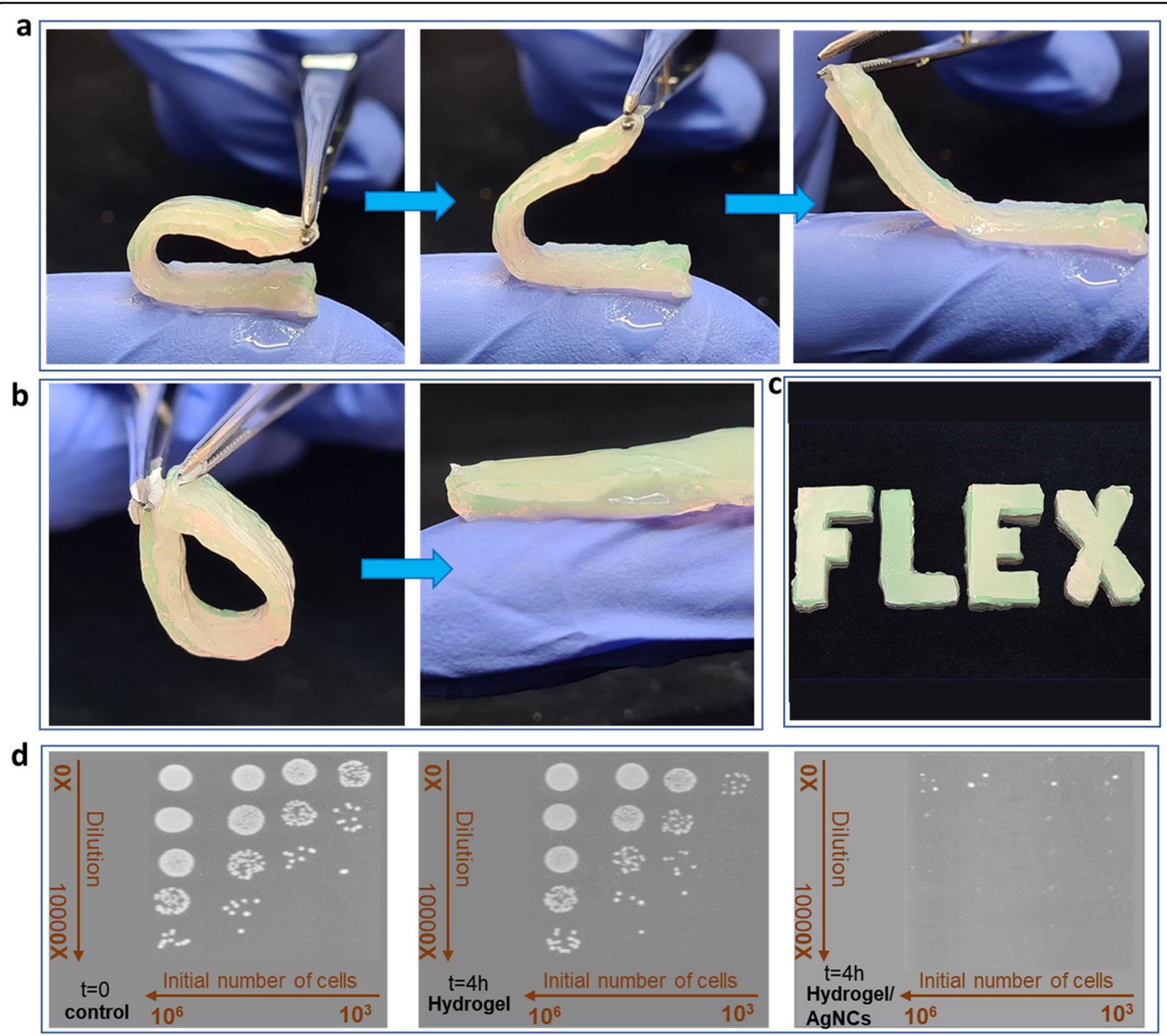

Fig. 4 Mechanical and antibacterial properties of the fabricated platform. a Macroscopic images of the platform folded and bent at $135^{\circ}$ and $45^{\circ}$, showing the platform's flexibility. $\mathbf{b}$ Macroscopic images of the fabricated platform folded and relaxed, indicating its ability to recover its original shape. c Macroscopic image of the platform cut into different shapes. $\mathbf{d}$ Photograph of bacteria in control samples at time 0 (left) and recovered after $4 \mathrm{~h}$ of incubation in the presence of hydrogel (middle) and hydrogel embedded with AgNCs (right). Only single colonies were recovered from samples incubated with hydrogel AgNCs, demonstrating the potent antibacterial effect of the silver particles.

exception; thus, the high swelling ratio of PNIPAAmbased hydrogels results in very poor physical strength ${ }^{64}$. This feature may not cause problems in applications in an aqueous state, but it is a major hurdle for applications such as biosensing, tissue engineering, and robotics ${ }^{65}$. To overcome this limitation, we introduced a nanofibrous mat into the system so that its coupling with the hydrogel could boost the mechanical properties of the final system. The fabricated platform showed outstanding mechanical properties and flexibility, enabling it to be folded and bent at different angles without causing any material structural damage. Macroscopic images of the platform folded and bent to $135^{\circ}$ and $45^{\circ}$ are shown in Fig. 4a. Furthermore, due to its flexibility, the platform can recover its original shape and size without any fractures from both physical and structural standpoints. Macroscopic images of the platform before and after removing a load are shown in Fig. 4b. This platform can be shaped into different designs, thus obtaining whatever shape is needed for other applications. A first example is shown in Fig. 4c, and another is presented in Figure S9. Mechanical tensile testing was also performed on the samples. Specimens were cut into the shape of dumbbells, placed between the grips of the mechanical testing machine, and subjected to 
an extension rate of $5 \mathrm{~mm} \mathrm{~min}^{-1}$. Figure S10 shows the sample's extension diagram, indicating the system's capacity for an extension up to $160 \%$ of its initial length. Additionally, the tensile strength of the specimens was calculated using Eq. 1. The tensile strength of the sample was calculated to be $0.32 \mathrm{~N} \mathrm{~mm}^{-1}$, which appears reasonable for the system.

$$
\text { Tensile strength }=\frac{\text { max load }}{\text { SA }}
$$

where SA is the surface area of the sample.

Thus, fibers are integrated into the hydrogel network by compositing a layer of electrospun fibers between two hydrogel layers. This procedure results in a compact, stable, and easily handled platform.

\section{Antibacterial properties of the composite platform}

Silver compounds such as silver nitrate and silver sulfadiazine have been used to prevent bacterial growth in applications such as drinking water decontamination and burn care ${ }^{66}$. Nanosilver is increasingly being introduced as an antibacterial agent in health care products ${ }^{67,68}$. Nanocrystalline silver provides a very large specific surface area for the release of ionic silver, and even a small amount of silver can provide potent bactericidal action ${ }^{69}$. Metallic silver has also been added to polymer fabrics and catheters to provide antibacterial properties ${ }^{70}$.

The antibacterial activity of the developed composite embedded with AgNCs was investigated by seeding Staphylococcus aureus (S. aureus) cells in phosphate-buffered saline (PBS) onto the sample surface. The bacterial cells were enumerated by serial dilutions at time zero and after $4 \mathrm{~h}$ of incubation. Figure $4 \mathrm{~d}$ illustrates colonies grown from serial dilutions of bacteria at time zero and after four hours of incubation on the hydrogel samples without silver nanocubes and samples with $20 \mathrm{wt} \%$ AgNCs. As shown in the images, after $4 \mathrm{~h}$ of incubation on the surface of the platform, only single colonies could be recovered, while numerous bacteria were grown after incubation on the surface of samples without AgNCs. Taking these results into account, it was calculated that after $4 \mathrm{~h}$ of incubation, half of the initial number of bacterial cells was recovered from neat hydrogels, while silver-containing hydrogels eliminated over $99.9 \%$ of bacteria independent of the AgNC concentration (Supplementary Fig. S11).

It is possible to assess that the presence of AgNCs in the platform can provide a powerful antibacterial effect to the multifunctional platform. As the antibacterial properties of silver are well known, the reduced number of bacteria on P(NIPAAm-co-NIPMAAm)/AgNC composites after a relatively short incubation time $(4 \mathrm{~h})$ confirms the presence and successful embedding of AgNCs into the hydrogel structure.

\section{Fast photothermal responsiveness of the composite}

The thermoresponsive behavior of the plasmonic nanoplatform was investigated using a green laser operating at $532 \mathrm{~nm}$ as a function of the laser intensity (from $1.43 \mathrm{~W}$ $\mathrm{cm}^{-2}$ to $15.71 \mathrm{~W} \mathrm{~cm}^{-2}$ ). AgNCs show an intense absorption band in the visible region, and owing to their remarkable photothermal efficiency, the absorbed light can be converted into heat ${ }^{71}$. The AgNCs embedded in the final composite are also responsible for the intense absorption band in the visible region of the final nanostructured platform. When the heat from the converted energy from green laser irradiation at the absorption peak exceeds the hydrogel VPTT, a cascade-like series of stimulus/response events leads to a rapid shrinkage of the hydrogel and water eviction. These phenomena demonstrate the hydrogel's significant thermoresponsive properties.

Figure $5 \mathrm{a}, \mathrm{b}$ show some thermographic images of the different hydration states and irradiation times of the platform after exposure to the laser beam at an intensity of $15.71 \mathrm{~W} \mathrm{~cm}^{-2}$. Images taken with the thermal camera detected an immediate increase in the temperature of the platform, which is considered the direct rate of responsiveness. Although the neat P(NIPAAm-co-NIPMAAm)based hydrogel has negligible photothermal responsivity (Fig. S12), plasmonic AgNCs make the composite hydrogel strongly responsive to visible light. As reported in Fig. 5a, exposure to laser irradiation for $70 \mathrm{~s}$ increased the temperature of dry samples to $\sim 80^{\circ} \mathrm{C}$; the hydrated hydrogel tested under the same conditions reached $\sim 50^{\circ} \mathrm{C}$. This difference is because the absorption peak varies, passing from hydrated to dry states and shifting the absorption peak in the case of a fully hydrated state (no longer centered on laser emissions), thus making the photoresponsivity less efficient. The different surrounding environments can also explain this phenomenon since air takes less time to reach high temperatures than water. Time-temperature profiles at different intensity values of the laser pump beam (from $1.43 \mathrm{~W} \mathrm{~cm}^{-2}$ to $15.71 \mathrm{~W}$ $\mathrm{cm}^{-2}$ ) are shown in Fig. $5 \mathrm{c}$, d. When the laser was ON, the temperature increased to almost $80^{\circ} \mathrm{C}$ in the dry state (Fig. 5c) and up to $50^{\circ} \mathrm{C}$ in the hydrated platform (Fig. $5 \mathrm{~d})$. In both cases, the temperature increase is attributed to the photothermal efficiency of AgNCs. When the laser was turned OFF, both samples cooled to room temperature in less than one minute. This behavior ensures the reusability of the system, as by increasing the temperature, the platform shrinks due to the LSCT nature of the hydrogel and expels the infiltrated solution. Removing the light source returns the hydrogel to its original state, and it is ready to be used again. A higher laser power could certainly lead to a faster heating efficiency of the sample. The linear correlation between the temperature and intensity of the laser can be seen in Fig. 5e, $\mathrm{f}$ for both hydrated and dry samples, respectively. Figure $5 \mathrm{~g}$ shows 

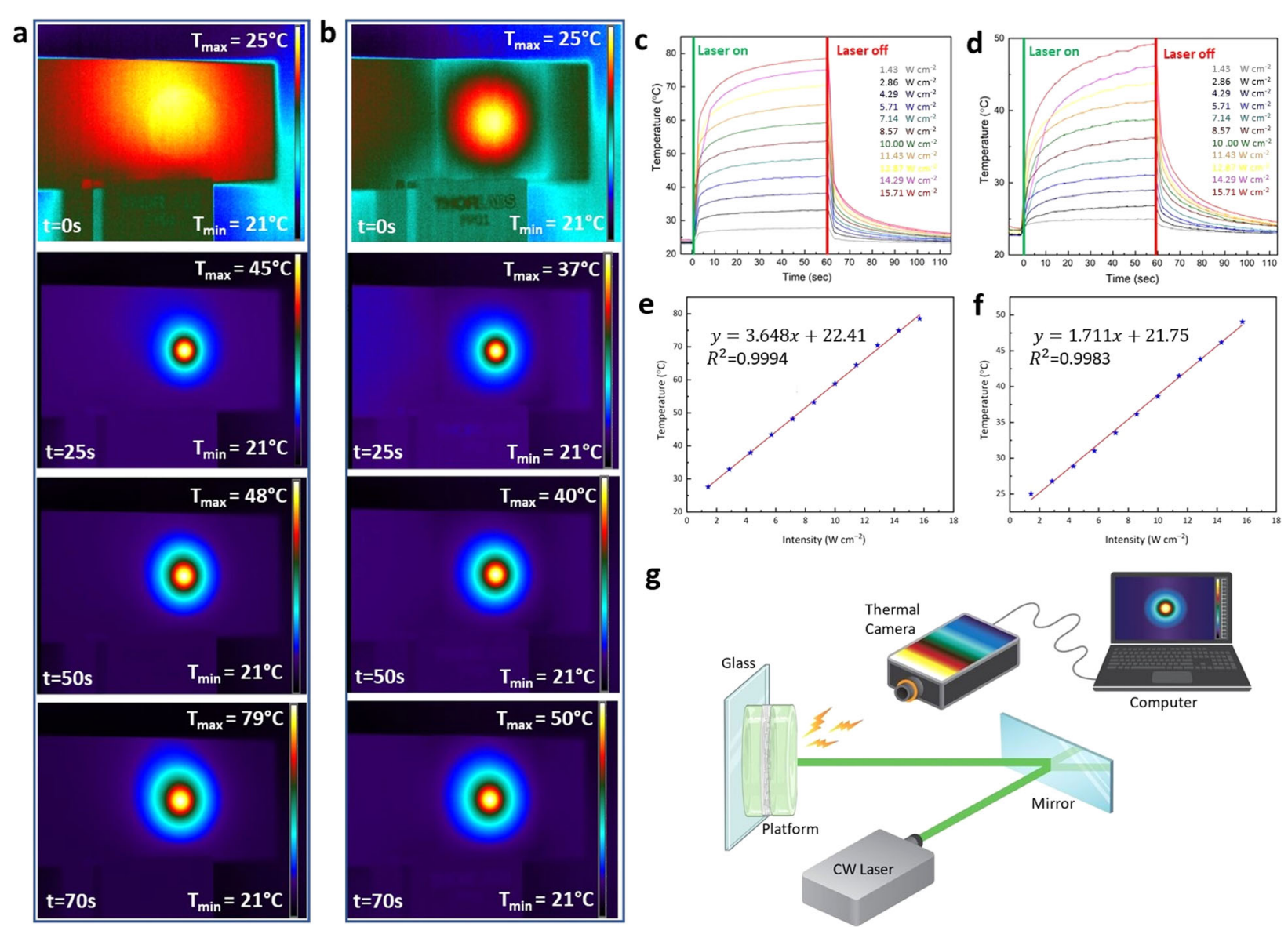

g

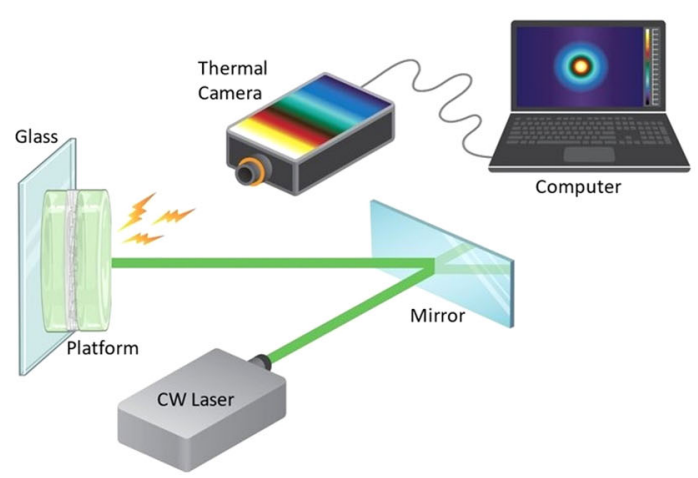

Fig. 5 Photothermal properties of the nanostructured platform demonstrating the fast responsiveness of the fabricated platform. a Thermographic images captured at different time points during laser irradiation (intensity: $15.57 \mathrm{~W} \mathrm{~cm}^{-2}$ ) of the platform fixed onto a glass slide in a dry state. At time zero, the platform and surrounding environment showed a similar temperature; at different time points, it is seen that the temperature increased to almost $80^{\circ} \mathrm{C}$. $\mathbf{b}$ Thermographic images captured at different time points during laser irradiation (intensity: $15.71 \mathrm{~W} \mathrm{~cm}{ }^{-2}$ ) of the platform fixed onto a glass slide in the hydrated state. At time zero, the platform and surrounding environment showed a similar temperature; at different time points, it is seen that the temperature increased to $50^{\circ} \mathrm{C}$. c Temperature-time plots for various intensity values (from $1.43 \mathrm{~W} \mathrm{~cm}{ }^{-2}$ to $15.71 \mathrm{~W} \mathrm{~cm}^{-2}$ ) of the laser beam in the dry state: the temperature increases due to the capability of AgNCs to efficiently convert the incident light into heat energy. $\mathbf{d}$ Temperature-time plots of the platform in the hydrated state according to various intensities of the laser beam: AgNC causes heat generation by efficiently converting incident light into heat energy. e Linear correlation between the maximum temperature detected and the intensity of the laser beam in the dry state. $\mathbf{f}$ Demonstration of the maximum temperature detected, which is linearly correlated with the intensity of the laser beam in the hydrated state of the platform. $\mathbf{g}$ Scheme showing the experimental setup designed to analyze the developed photothermal properties of the platform: the laser beam running from top to bottom while irradiating the platform was observed with a thermal camera that records images and transfers them directly to a computer.

the experimental optical setup used for photothermal investigation of the platforms. A continuous-wave $(\mathrm{CW})$ laser source operating at $532 \mathrm{~nm}$ irradiated the samples placed in a tailor-designed specimen holding the nanostructured platform after passing through a customdesigned optical patch. A side-view thermal camera recorded thermographic images with changes in the sample temperature at different irradiation times.

\section{Glucose sensing}

According to optical transducer/phenomena, optical glucose sensors are classified into four groups: surface plasmon resonance (SPR), fluorescent, surface-enhanced Raman scattering (SERS), and photonic bandgap sensors. Our multifunctional platforms were investigated for glucose sensing due to their high sensitivity to changes in the surrounding refractive index. The readout of these sensors depended on the change detected in the resonant absorbed wavelength with glucose concentrations. Localized surface plasmon resonance (LSPR) is based on the surface plasmon resonance phenomenon in which metal electrons oscillate coherently in an electromagnetic field during surface conduction. This phenomenon can enhance the measurement sensitivity of the local environment on the metal nanostructure surface. 
To first examine the sensing reliability of the AgNCs, nanocubes were tested dispersed in liquid solutions with different glucose concentrations. The shift of the wavelength of the main LSPR band can be seen in Fig. S13, which shows that the AgNCs are sensitive to glucose. Biosensors using plasmonic NPs have been mainly developed using techniques such as electrodeposition of nanoparticles to functionalize the surface of supporting materials. Although this technique allows good attachment of particles on the surface, the immobilization of nanoparticles on the supporting material surface greatly reduces the contact area between the nanomaterials and the tested analyte. To overcome this drawback, the incorporation of plasmonic nanoparticles into bulk hydrogels has been explored. Indeed, embedding nanoparticles into the hydrogel mass renders the entire nanoparticle surface available for exposure to the sample. This elegant strategy strongly improves the sensor sensitivity. In addition, particle encapsulation is an inexpensive and simple approach compared to the electrodeposition of nanoparticles.

Figure 6a shows a schematic illustration of the glucosesensing procedure in human urine samples. As shown in this scheme and described in the materials and methods section, the analyzed human urine sample was added to various standard glucose solutions and infiltrated into the hydrogel/mat platform. The hydrogel samples were placed in a UV-Vis spectrophotometer, and the shift of the maximum LSPR band was detected.

Figure $6 \mathrm{~b}$ shows the absorption spectra of P(NIPAAmco-NIPMAAm)/AgNCs in three different infiltrating media: air, pure water, and an $8 \mathrm{mM} \mathrm{D}$-glucose solution. The absorption spectrum of the composite in air shows an intense plasmonic peak centered at $551 \mathrm{~nm}$, which is associated with the resonance of the (100) planes of AgNCs, along with two peaks at $410 \mathrm{~nm}$ and $352 \mathrm{~nm}$, attributed to the edges and corners of AgNCs, respectively $^{72}$. P(NIPAAm-co-NIPMAAm) hydrogels possess a refractive index $(n=1.5)$ higher than that of water $(n=$ 1.3). Therefore, the position of the plasmonic peaks of the hydrogel samples embedded with AgNCs is shifted with respect to the plasmonic peaks of the colloidal dispersion of AgNCs (shown in Fig. 2c).

The infiltration of the P(NIPAm-co-NIPMAm)/AgNC nanocomposite with Milli-Q water resulted in a blueshift of $37 \mathrm{~nm}\left(\Delta \lambda_{T}\right)$ from the main plasmonic peak of AgNCs, as highlighted by the dark blue track in Fig. 6b. In comparison, a lower blueshift $(31 \mathrm{~nm})\left(\Delta \lambda_{\mathrm{S}}\right)$ was measured following the infiltration of P(NIPAAm-co-NIPMAAm)/ AgNC samples with an $8 \mathrm{mM}$ D-glucose solution.

The spectral shift of the absorption spectrum of $\mathrm{P}$ (NIPAAm-co-NIPMAAm)/AgNC samples is due to variation in the refractive index surrounding AgNCs; the average refractive index is expected to decrease slightly after Milli-Q water is introduced into the dried $P$ (NIPAAm-co-NIPMAAm) sample ${ }^{73}$. Accordingly, the decreased local refractive index value resulted in a blueshift of the plasmon bands of AgNCs in the P(NIPAAmco-NIPMAAm)/AgNC sample. This significant $\Delta \lambda_{\mathrm{T}}$ value can be further elucidated considering that the infiltration of P(NIPAAm-co-NIPMAAm)/AgNCs with Milli-Q water can alter the arrangement of the P(NIPAAm-co-NIPMAAm) molecules surrounding AgNCs. In particular, the $\mathrm{P}$ (NIPAAm-co-NIPMAAm) structure can change from a collapsed state (when the polymer is dry) to a swollen state following infiltration with Milli-Q water. Indeed, water is an optimal solvent for P(NIPAAm-co-NIPMAAm), as it minimizes attractive forces among polymer molecules, thus promoting polymer swelling. In the swollen state, the density of the polymer molecules embedded with AgNCs is lower than that of P(NIPAAmco-NIPMAAm) molecules in the dried state. The lower density in the swollen state can reduce the refractive index value for P(NIPAAm-co-NIPMAAm), as demonstrated by Tagliazucchi et al. ${ }^{74}$. Therefore, when the P(NIPAAm-coNIPMAAm) sample was infiltrated with water, the density of the polymer molecules surrounding AgNCs decreased, leading to a consequent further decrease in the $\mathrm{P}$ (NIPAAm-co-NIPMAAm) refractive index value, thereby causing an intense blueshift of the AgNC plasmon band ${ }^{75}$.

When the plasmonic nanoplatform was infiltrated with an $8 \mathrm{mM}$ D-glucose solution, a less intense $(31 \mathrm{~nm})$ blueshift was measured. Indeed, the presence of D-glucose molecules in the infiltrating solution can produce an overall increase in the local refractive index value experienced by AgNCs (compared with Milli-Q water); this situation resulted in a less intense blueshift than the optical shift measured for the P(NIPAAm-co-NIPMAAm)/AgNC sample infiltrated with Milli-Q water.

Figure $6 \mathrm{c}$ shows the absorption spectra of the $\mathrm{P}$ (NIPAAm-co-NIPMAAm)/AgNC samples infiltrated with D-glucose standard solutions at different concentrations used to prepare a typical calibration curve and, in turn, to evaluate the limits of detection (LODs) of the system. As the $\mathrm{D}$-glucose concentration decreased in the infiltrating solution, the peak in the corresponding absorption spectrum of the infiltrated sample shifted toward lower wavelengths due to a reduced average refractive index. Moreover, it is well known that if the concentration of D-glucose decreases, the viscosity of the infiltrating solution is reduced ${ }^{76}$. Consequently, attractive interactions among P(NIPAAm-co-NIPMAAm) molecules decrease. Under these conditions, polymer swelling is progressively promoted (compared to polymer collapse), thus resulting in a progressively lower local refractive index value. This change results in a more intense blueshift of the AgNC plasmon band than that of the dry $\mathrm{P}$ (NIPAAm-co-NIPMAAm)/AgNC sample ${ }^{74}$. The ability of 
a
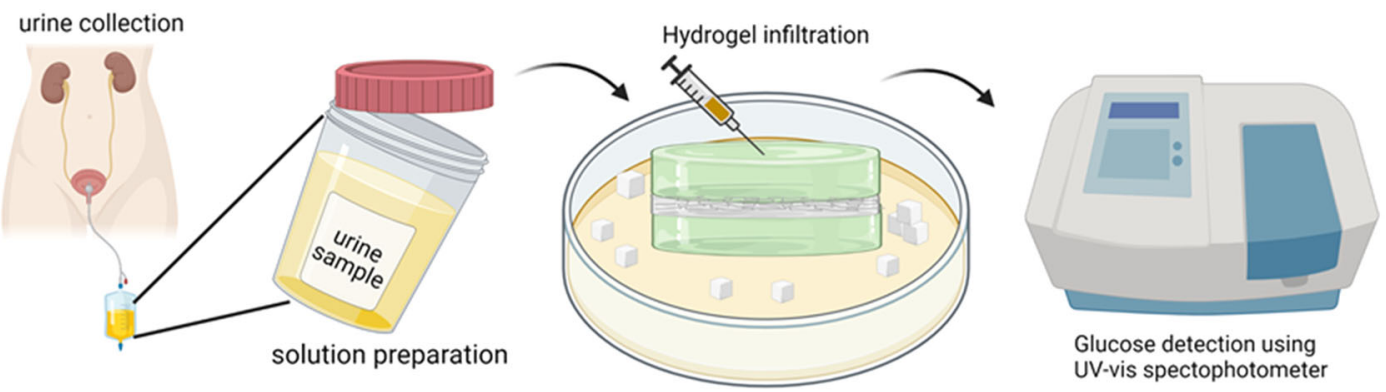

b
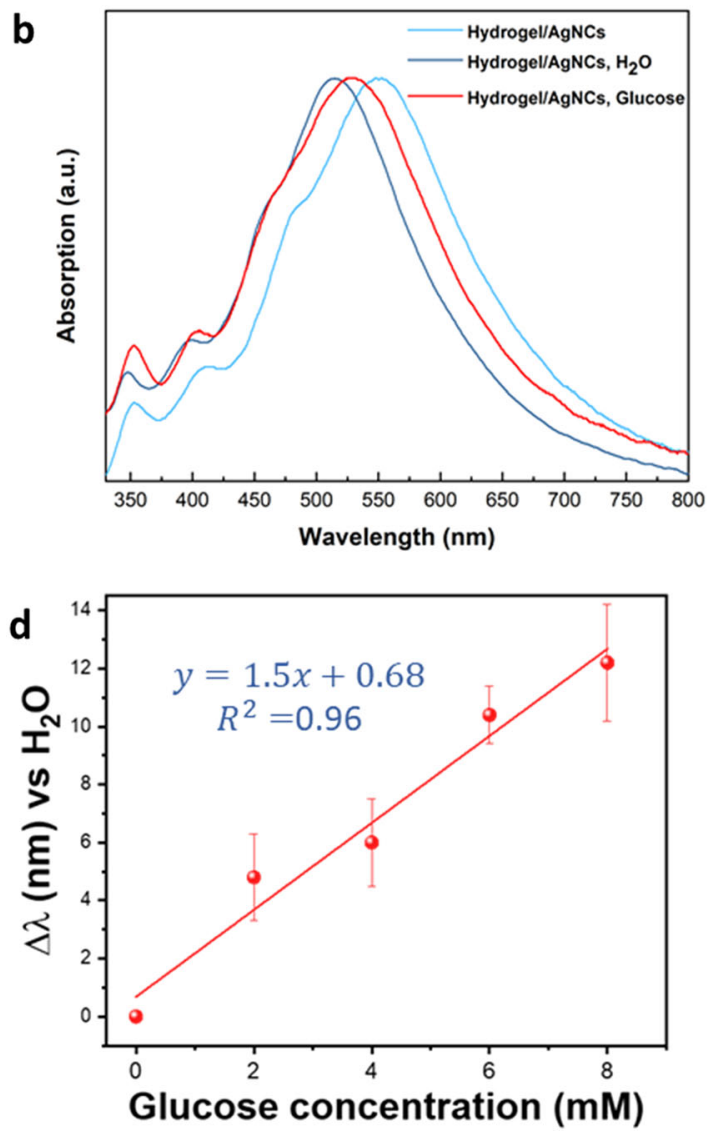

C

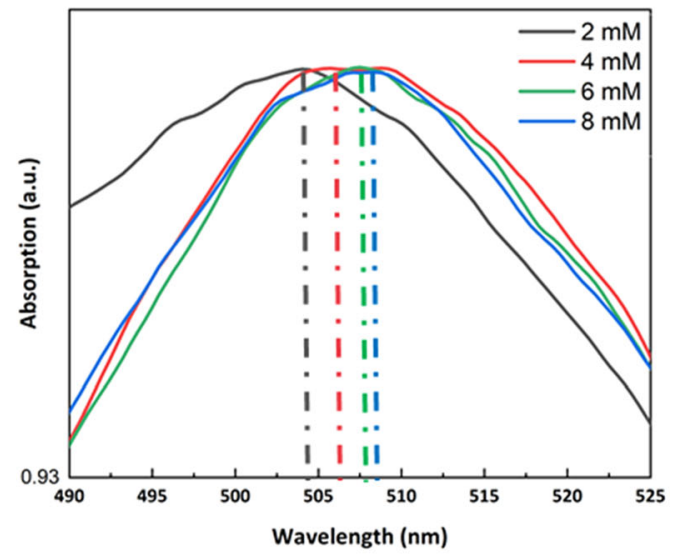

e

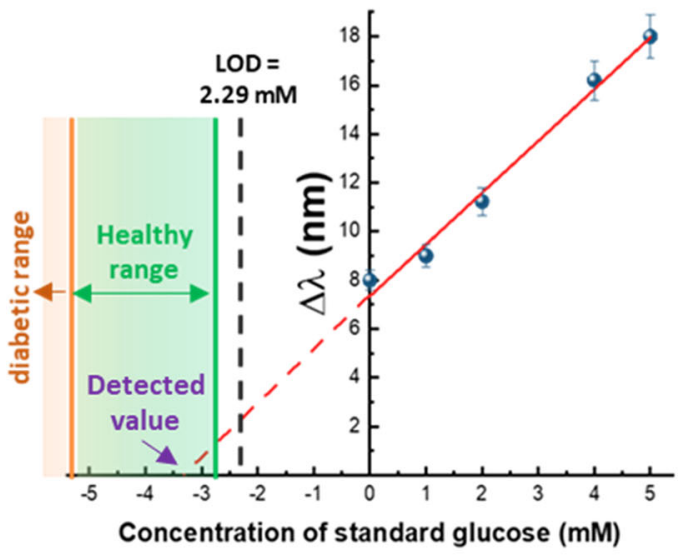

Fig. 6 Glucose sensing of the P(NIPAAm-co-NIPMAAm)/AgNC platform. a Scheme illustrating glucose quantification steps. Urine samples were collected from humans, the platform was infiltrated with a urine solution, and the amount of glucose available in the solution was measured using a UV-Vis spectrophotometer. b Normalized absorption spectrum of three representative P(NIPAm-co-NIPMAm)/AgNC samples in the dry state (light blue track) infiltrated with Milli-Q water (blue track) and an 8 mM D-glucose solution (red track). c Representative normalized absorption spectra of P (NIPAAm-Co-NIPMAAm)/AgNC samples infiltrated with standard solutions of D-glucose, highlighting variations in the optical response of the plasmonic nanoplatform as a function of the D-glucose concentration. $\mathbf{d}$ Plasmonic shift $(\Delta \lambda)$, shown as a function of glucose concentration for a set of standard glucose solutions and linear interpolation. $\Delta \lambda$ was evaluated as the difference between the maximum wavelength measured for the nanocomposite infiltrated with Milli-Q water and the maximum wavelength measured for the nanocomposite infiltrated with a standard solution of D-glucose. Using the LOD calculated from the curve, the platform's ability to sense glucose levels at both normal and diabetic levels was also indicated. e A representative determination of the D-glucose concentration in a human urine sample, determined from $\Delta \lambda$ values, using the standard addition method, confirming the platform's capability to be used as a glucose sensor.

P(NIPAAm-co-NIPMAAm)/AgNC hydrogels to behave as plasmonic-based refractive index sensors was also evaluated by associating the plasmonic shift with the concentration of D-glucose standard solutions. The calibration curve obtained as described earlier shows a linear correlation between the plasmonic shift $(\Delta \lambda)$ and $\mathrm{D}$-glucose concentration for the sensing of the platform (Fig. 6d). The limit of detection (LOD) of the system was calculated to be $2.29 \mathrm{mM}$, which is fully consistent with the typical detection limits of commercial urine strip 
tests ${ }^{46}$. This result emphasizes the sensitivity of $\mathrm{P}$ (NIPAAm-co-NIPMAAm)/AgNCs to variations in the local refractive index value. Indeed, based on the work published by Surendar et al. used to calculate the refractive index value of the D-glucose standard solutions ${ }^{77}$, we were able to estimate a sensitivity of $433 \mathrm{~nm} / \mathrm{RIU}$ (refractive index unit), which is also in agreement with the results previously reported in the literature for silver nanoparticles ${ }^{78}$.

Finally, we tested our sensor's ability to analyze the D-glucose concentration in human urine samples by exploiting the method of standard additions. This method was used because although urine samples are relatively simple, it enabled avoiding any matrix effect and further sample preparation before performing tests ${ }^{79}$. Additionally, it is worth mentioning that we avoided testing artificial urine in this protocol to verify the ability of the developed platform to test real human samples ${ }^{80}$. As previously described, hydrogel composite samples were infiltrated with D-glucose standard solutions, and absorption wavelengths were measured. As shown in Fig. $6 e$, with the increase in the concentration of standard D-glucose solutions, the plasmonic shift redshifted in response to the change in the local reflective index, as previously reported and discussed for standard D-glucose solutions, according to the experimental results. Figure $6 \mathrm{e}$ shows a representative analysis of the developed method based on the use of the nanostructured platform, which permitted detection of a D-glucose amount of $3.7 \pm$ $0.3 \mathrm{mM}$ in the investigated sample. This technique was explored to detect glucose in urine as a proof-of-concept of the proposed noninvasive detection method, showing excellent detection of glucose levels within a wide range of concentrations. The glucose level in healthy people is between 2.78 and $5.5 \mathrm{mM}$ and more than $5.5 \mathrm{mM}$ for diabetic people ${ }^{81}$. This range of glucose concentrations is above the detection limit calculated by our system, which is reliable and reproducible considering the calculated standard deviation. All these results prove the sensor's ability to detect the desired glucose levels in urine. It is worth mentioning that the reusability of the platform is ensured by the photothermal responsivity of the system, which expels the liquid infiltrating the gel upon irradiation.

\section{Conclusions}

In this work, we successfully fabricated an antibacterial photothermal-responsive glucose-sensing platform inspired by the structure of chameleon skin. The multifunctional platform consists of a layer of $\mathrm{PEO} / \mathrm{PCL}$ electrospun fibers enclosed by two layers of P(NIPAAm-coNIPMAAm)-based hydrogels with embedded plasmonic nanoparticles, i.e., AgNCs with outstanding sensing performance. The P(NIPAAm-co-NIPMAAm) hydrogel is a thermoresponsive hydrogel. Coupling this hydrogel with AgNCs made it possible to develop novel and unique functions of the platform. AgNCs endow the platform with antibacterial properties, promote the hydrogel's photothermal responsivity with plasmonic properties and, most importantly, enable the system's sensing feature. Several studies were performed using laser beam irradiation at $532 \mathrm{~nm}$ and a high-resolution thermal camera to demonstrate the photothermal behavior of the proposed platform. The presence of a PEO/PCL nanofibrous mat was a requisite for mechanical stability of the system. Indeed, hydrogel itself consists of $94 \%$ water and does not have suitable mechanical stability and physical properties for handling as a platform for sensing applications. The $\mathrm{PEO} / \mathrm{PCL}$ nanofibrous mat gives the platform excellent mechanical properties to allow the system to be designed in various shapes and overcome mechanical failures during bending, twisting, and stretching, thus ensuring that the structure and uniformity of the platform are maintained. The presence of AgNCs imparted plasmonic properties to the proposed nanoplatform. The wellstructured platform was tested as a biosensor to detect glucose in body fluids, presenting an accurate and reproducible procedure. The platform provided a fast and cost-effective glucose monitoring procedure as an LSPR sensor and was successfully tested for analysis of glucose in a human urine sample by following an accurate and reproducible procedure. The detection limit of this platform $(2.29 \mathrm{mM})$ is lower than the glucose levels in both healthy and diabetic people; additionally, this method benefits from the fact that it is noninvasive. Optical detection is based on the difference in the refractive index of the surrounding environment with a linear coloration between the $\Delta \lambda$ and glucose concentration in urine samples. The presence of glucose causes a change in the reflective index of the surrounding medium, thus producing a shift in absorption peaks. Considering the platform's antibacterial properties and photothermal responsiveness, along with the sensing capability, our system has been proven to be a promising platform capable of detecting glucose levels in body fluids.

\footnotetext{
Acknowledgements

This study was partially supported by the First TEAM grant number POIR.04.04.00-00-5ED7/18-00, which is carried out within the framework of the First TEAM program of the Foundation for Polish Science (FNP), co-financed by the European Union under the European Regional Development Fund. The authors are also grateful for the support of this work by the National Agency for Academic Exchange (NAWA), grant no. PPI/APM/2018/1/00045/U/001. Chiara Rinoldi, Paweł Nakielski, and Filippo Pierini acknowledge financial support from the Polish Ministry of Science and Higher Education through scholarships for outstanding young scientists. Chiara Rinoldi was partially supported by the Foundation for Polish Science (FNP). Luciano De Sio acknowledges financial support from the Air Force Office of Scientific Research (AFOSR), Air Force Research Laboratory (AFRL) and U.S. Air Force grant no. FA9550-18-1-0038 (P.I.L. De Sio, EOARD 2017-2020) and the Materials and Manufacturing; the Sapienza University of Rome, under grant no. RM11816431206A2C (Progetto di Ateneo 2018, P.I.L. De Sio). Part of this
} 
research was also carried out using apparatuses available thanks to EC structural funds within the framework of the Center for Preclinical Research and Technology (CePT), POIG No. 02.02.00-17-024/08-00.

\section{Author details}

'Department of Biosystems and Soft Matter, Institute of Fundamental Technological Research, Polish Academy of Sciences, Warsaw 02-106, Poland. ${ }^{2}$ Institute of Crystallography CNR-IC, National Research Council of Italy, Via Salaria Km 29,300, 00015 Monterotondo - Rome, Italy. ${ }^{3}$ Mossakowski Medical Research Institute, Polish Academy of Sciences, 02-106 Warsaw, Poland. ${ }^{4}$ Innovation Center for Textile Science and Technology, Donghua University, West Yan'an Road 1882, Shanghai 200051, China. ${ }^{5}$ Department of MedicoSurgical Sciences and Biotechnologies - Research Center for Biophotonics, Sapienza University of Rome Corso della Repubblica 79, 04100 Latina, Italy. ${ }^{6}$ CNR-Lab. Licryl, Institute NANOTEC, Arcavacata di Rende 87036, Italy

\section{Author contributions}

Y.Z. fabricated the nanoplatforms, performed the structural and mechanical characterization, and wrote the article. F.Pe. carried out the biosensing tests. C. R. developed the nanoplatforms. P.N. performed the platform structural characterization. A.Z. fabricated the platform and carried out the structural characterization. T.A.K. performed the platform structural characterization. W.A. conducted the antibacterial studies. X.L. carried out the mechanical tests. A.C. performed the nanoplatform optical characterization. I.S. carried out the antibacterial test. B.D. conducted the mechanical characterization. L.D.S formulated the idea and conducted the photoresponsiveness experiments. F. Pi. conceived the idea, designed the experiments, and supervised the project. All authors discussed the results and revised the manuscript.

\section{Data availability}

The data that support the findings of this study are available from the corresponding author upon reasonable request.

\section{Conflict of interest}

The authors declare no competing interests.

\section{Publisher's note}

Springer Nature remains neutral with regard to jurisdictional claims in published maps and institutional affiliations.

Supplementary information The online version contains supplementary material available at https://doi.org/10.1038/s41427-022-00365-9.

Received: 29 October 2021 Revised: 31 December 2021 Accepted: 12 January 2022.

Published online: 4 March 2022

\section{References}

1. Katiyar, N., Goel, G., Hawi, S. \& Goel, S. Nature-inspired materials: emerging trends and prospects. NPG Asia Mater. 3, 56 (2021).

2. Perera, A. S. \& Coppens, M.-O. Re-designing materials for biomedical applications: from biomimicry to nature-inspired chemical engineering. Trans. $R$. Soc. A 377, 20180268 (2019)

3. Kimovski, D., ljaz, H., Saurabh, N. \& Prodan, R. Adaptive nature-inspired fog architecture. 2018 IEEE 2nd International Confere Washington, DC, 1-3 May 2018.

4. Pfeifer, R., Lungarella, M. \& lida, F. Self-organization, embodiment, and biologically inspired robotics. Science 318, 1088-1093 (2007).

5. Chen, J. et al. Nature-inspired hierarchical protrusion structure construction for washable and wear-resistant superhydrophobic textiles with self-cleaning ability. ACS Appl. Mater. Interfaces 13, 18142-18151 (2021).

6. $\mathrm{Xu}, \mathrm{C}$. et al. Nature-inspired hierarchical materials for sensing and energy storage applications. Chem. Soc. Rev. 50, 4856-4871 (2021).

7. Xu, K, Lu, Y. \& Takei, K. Multifunctional skin-inspired flexible sensor systems for wearable electronics. Adv. Mater. Technol. 4, 1800628 (2019).
8. Goel, G., Hélix-Nielsen, C., Upadhyaya, H. M. \& Goel, S. A bibliometric study on biomimetic and bioinspired membranes for water filtration. NPJ Clean. Water 4, 41 (2021).

9. Zhu, C. et al. A nature-inspired, flexible substrate strategy for future wearable electronics. Small 15, 1-11 (2019).

10. Lee, S.W. et al. NiCHE platform: nature-inspired catechol-conjugated hyaluronic acid environment platform for salivary gland tissue engineering. ACS Appl. Mater. Interfaces 12, 4285-4294 (2020).

11. Dong, Y. et al. Chameleon-inspired strain-accommodating smart skin. ACS Nano 13, 9918-9926 (2019).

12. Wang, Y., Cui, H., Zhao, Q. \& Du, X. Chameleon-inspired structural-color actuators. Matter 1, 626-638 (2019).

13. Chou, H.H. et al. A chameleon-inspired stretchable electronic skin with interactive colour changing controlled by tactile sensing. Nat. Commun. $\mathbf{6}$, 1-10 (2015).

14. Lee, G. H. et al. Chameleon-inspired mechanochromic photonic films composed of non-close-packed colloidal arrays. ACS Nano 11, 11350-11357 (2017)

15. Teyssier, J., Saenko, S., van der Marel, D. \& Milinkovitch, M. C. Photonic crystals cause active colour change in chameleons. Nat. Commun. 6, 6368 (2015).

16. Kergoat, L., Piro, B., Berggren, M., Horowitz, G. \& Pham, M. C. Advances in organic transistor-based biosensors: From organic electrochemical transistors to electrolyte-gated organic field-effect transistors. Anal. Bioanal. Chem. 402, 1813-1826 (2012).

17. Sun, C. et al. Preparation of novel electrochemical glucose biosensors for whole blood based on antibiofouling polyurethane-heparin nanoparticles. Electrochim. Acta 97, 349-356 (2013).

18. Bruen, D., Delaney, C., Florea, L. \& Diamond, D. Glucose sensing for diabetes monitoring: recent developments. Sensors 17, 1866 (2017).

19. Wang, J. Electrochemical Glucose Biosensors. Chem. Rev. 108, 814-825 (2008).

20. Chen, C. et al. Recent advances in electrochemical glucose biosensors: a review. RSC Adv. 3, 4473-449 (2013).

21. Nery, E. W., Kundys, M., Jeleń, P. S. \& Jönsson-Niedziólka, M. Electrochemical glucose sensing: Is there still room for improvement? Anal. Chem. 88, 11271-11282 (2016)

22. Mcnichols, R. J. \& Coté, G. L. Optical glucose sensing in biological fluids: an overview. J. Biomed. Opt. 5, 5-16 (2000).

23. Makaram, P., Owens, D. \& Aceros, J. Trends in nanomaterial-based non-invasive diabetes sensing technologies. Diagnostics 4, 27-46 (2014).

24. Lee, $\mathrm{H}$. et al. Wearable/disposable sweat-based glucose monitoring device with multistage transdermal drug delivery module. Sci. Adv. 3, e1601314 (2017).

25. Zhang, W., Du, Y. \& Wang, M. L. On-chip highly sensitive saliva glucose sensing using multilayer films composed of single-walled carbon nanotubes, gold nanoparticles, and glucose oxidase. Sens. Bio-Sens. Res. 4, 96-102 (2015).

26. Farandos, N. M., Yetisen, A. K., Monteiro, M. J., Lowe, C. R. \& Yun, S. H. Contact lens sensors in ocular diagnostics. Adv. Healthc. Mater. 4, 792-810 (2015).

27. Saraoglu, H. M. \& Mehmet, K. Determination of blood glucose level-based breath analysis by a quartz crystal microbalance sensor array. IEEE Sens. J. 10 104-109 (2010).

28. Elsherif, M., Hassan, M. U., Yetisen, A. K. \& Butt, H. Hydrogel optical fibers for continuous glucose monitoring. Biosens. Bioelectron. 137, 25-32 (2019).

29. Bartic, C. \& Borghs, G. Organic thin-film transistors as transducers for (bio) analytical applications. Anal. Bioanal. Chem. 384, 354-365 (2006).

30. Wang, L., Fine, D., Sharma, D., Torsi, L. \& Dodabalapur, A. Nanoscale organic and polymeric field-effect transistors as chemical sensors. Anal. Bioanal. Chem. 384, 310-321 (2006)

31. Ahmed, E. M. Hydrogel: Preparation, characterization, and applications: A review. J. Adv. Res. 6, 105-121 (2015).

32. Kopeček, J. Hydrogel biomaterials: A smart future? Biomaterials 28, 5185-5192 (2007).

33. Kamath, K. R. \& Park, K. Biodegradable hydrogels in drug delivery. Adv. Drug Deliv. Rev. 11, 59-84 (1993).

34. Vashist, A., Vashist, A., Gupta, Y. K. \& Ahmad, S. Recent advances in hydrogel based drug delivery systems for the human body. J. Mater. Chem. B 2, 147-166 (2014).

35. Sood, N., Bhardwaj, A., Mehta, S. \& Mehta, A. Stimuli-responsive hydrogels in drug delivery and tissue engineering. Drug Deliv. 23, 748-770 (2016).

36. Kim, D., Kim, H., Lee, E., Jin, K. S. \& Yoon, J. Programmable volume phase transition of hydrogels achieved by large thermal hysteresis for static-motion Bilayer Actuators. Chem. Mater. 28, 8807-8814 (2016). 
37. Xue, Q. et al. Nanostrip flexible microwave enzymatic biosensor for noninvasive epidermal glucose sensing. Nanoscale Horiz. 5, 934-943 (2020).

38. Ruel-Gariépy, E. \& Leroux, J. C. In situ-forming hydrogels - Review of temperature-sensitive systems. Eur. J. Pharm. Biopharm. 58, 409-426 (2004).

39. Nakielski, P. et al. Multifunctional platform based on electrospun nanofibers and plasmonic hydrogel: a smart nanostructured pillow for near-infrared lightdriven biomedical applications. ACS Appl. Mater. Interfaces 12, 54328-54342 (2020).

40. Heskins, M. \& Guillet, J. E. Solution Properties of Poly(N-isopropylacrylamide). J. Macromol. Sci. Part A - Chem. 2, 1441-1455 (1968).

41. Haq, M. A., Su, Y. \& Wang, D. Mechanical properties of PNIPAM based hydrogels: A review. Mater. Sci. Eng. C. 70, 842-855 (2017).

42. Anker, J. N., et al. in Nanosci. Technol. A Collect. Rev. from Nat. Journals 308-319, https://doi.org/10.1142/9789814287005_0032 (World Scientific Publishing Co., 2009).

43. Sannomiya, T. \& Vörös, J. Single plasmonic nanoparticles for biosensing. Trends Biotechnol. 29, 343-351 (2011).

44. Guglielmelli, A. et al. Thermoplasmonics with gold nanoparticles: a new weapon in modern optics and biomedicine. Adv. Photonics Res. 2, 2000198 (2021).

45. Dreaden, E. C., Alkilany, A. M., Huang, X., Murphy, C. J. \& El-Sayed, M. A. The golden age: gold nanoparticles for biomedicine. Chem. Soc. Rev. 41, 2740-2779 (2012).

46. Loiseau, A. et al. Silver-based plasmonic nanoparticles for and their use in biosensing. Biosensors 9, 78 (2019).

47. Narayanan, R. \& El-Sayed, M. A. Changing catalytic activity during colloidal platinum nanocatalysis due to shape changes: Electron-transfer reaction. J. Am. Chem. Soc. 126, 7194-7195 (2004).

48. Lee, C. L., Chang, K. C. \& Syu, C. M. Silver nanoplates as inkjet ink particles for metallization at a low baking temperature of $100^{\circ} \mathrm{C}$. Colloids Surf. A Physicochem. Eng. Asp. 381, 85-91 (2011).

49. He, M., Wang, Q., Zhang, J., Zhao, W. \& Zhao, C. Substrate-independent Agnanoparticle-loaded hydrogel coating with regenerable bactericidal and thermoresponsive antibacterial properties. ACS Appl. Mater. Interfaces 9 , 44782-44791 (2017)

50. Lannutti, J., Reneker, D., Ma, T., Tomasko, D. \& Farson, D. Electrospinning for tissue engineering scaffolds. Mater. Sci. Eng. C. 27, 504-509 (2007).

51. Sill, T. J. \& von Recum, H. A. Electrospinning: applications in drug delivery and tissue engineering. Biomaterials 29, 1989-2006 (2008).

52. Liu, M., Duan, X.P., Li, Y.-M., Yang, D.-P. \& Long, Y.-Z. Electrospun nanofibers for wound healing. Mater. Sci. Eng. C. 76, 1413-1423 (2017).

53. Ren, G. et al. Electrospun poly(vinyl alcohol)/glucose oxidase biocomposite membranes for biosensor applications. React. Funct. Polym. 66, 1559-1564 (2006).

54. Li, D., Frey, M. W. \& Baeumner, A. J. Electrospun polylactic acid nanofiber membranes as substrates for biosensor assemblies. J. Membr. Sci. 279, 354-363 (2006)

55. Liang, D., Hsiao, B. S. \& Chu, B. Functional electrospun nanofibrous scaffolds for biomedical applications. Adv. Drug Deliv. Rev. 59, 1392-1412 (2007).

56. Li, W. J., Laurencin, C. T., Caterson, E. J., Tuan, R. S. \& Ko, F. K. Electrospun nanofibrous structure: A novel scaffold for tissue engineering. J. Biomed. Mater. Res. 60, 613-621 (2002).

57. Yang, Q. et al. Highly sensitive and selective sensor probe using glucose oxidase/gold nanoparticles/graphene oxide functionalized tapered optical fiber structure for detection of glucose. Optik 208, 164536 (2020).

58. Rinoldi, C. et al. Mechanical and biochemical stimulation of 3D multilayered scaffolds for tendon tissue engineering. ACS Biomater. Sci. Eng. 5, 2953-2964 (2019).

59. Pawłowska, S. et al. Ultraviolet light-assisted electrospinning of core-shell fully cross-linked P(NIPAAm-Co-NIPMAAm) hydrogel-based nanofibers for thermally induced drug delivery self-regulation. Adv. Mater. Interfaces 7 2000247 (2020)

60. Rinoldi, C. et al. Three-dimensional printable conductive semi-interpenetrating polymer network hydrogel for neural tissue applications. Biomacromolecules 22, 3084-3098 (2021).

61. Nasajpour, A. et al. Cholesteryl ester liquid crystal nanofibers for tissue engineering applications. ACS Mater. Lett. 2, 1067-1073 (2020).

62. Baker, B. M. et al. The potential to improve cell infiltration in composite fiberaligned electrospun scaffolds by the selective removal of sacrificial fibers. Biomaterials 29, 2348-2358 (2008).

63. Kupka, V. et al. Well-blended PCL/PEO electrospun nanofibers with functional properties enhanced by plasma processing. Polymers 12, 1403 (2020).

64. Haq, M. A., Su, Y. \& Wang, D. Mechanical properties of PNIPAM based hydrogels: a review. Mater. Sci. Eng. C. 70, 842-855 (2017).

65. Yoon, C. et al. Functional stimuli responsive hydrogel devices by self-folding. Smart Mater. Struct. 23, 094008 (2014).

66. Praveena, S. M., Karuppiah, K. \& Than, L. T. L. Potential of cellulose paper coated with silver nanoparticles: a benign option for emergency drinking water filter. Cellulose 25, 2647-2658 (2018).

67. Chen, X. \& Schluesener, H. J. Nanosilver: a nanoproduct in medical application Toxicol. Lett. 176, 1-12 (2008).

68. Anjum, S. \& Gupta, B. Bioengineering of functional nanosilver nanogels for smart healthcare systems. Glob. Chall. 2, 1800044 (2018).

69. Fong, J. \& Wood, F. Nanocrystalline silver dressings in wound management: a review. Int. J. Nanomed. 1, 441-449 (2006).

70. Wei, D., Sun, W., Qian, W., Ye, Y. \& Ma, X. The synthesis of chitosan-based silver nanoparticles and their antibacterial activity. Carbohydr. Res. 344, 2375-2382 (2009).

71. Yin, Q. Q. et al. Plasmonic molybdenum oxide nanosheets supported silver nanocubes for enhanced near-infrared antibacterial activity: Synergism of photothermal effect, silver release and photocatalytic reactions. Appl. Catal. B 224, 671-680 (2018).

72. Wang, B., Zhang, L. \& Zhou, X. Synthesis of silver nanocubes as a SERS substrate for the determination of pesticide paraoxon and thiram. Spectrochim. Acta - Part A Mol. Biomol. Spectrosc. 121, 63-69 (2014).

73. Pierini, F. et al. Thermoplasmonic-activated hydrogel based dynamic light attenuator. Adv. Opt. Mater. 8, 2000324 (2020).

74. Tagliazucchi, M., Blaber, M. G., Schatz, G. C., Weiss, E. A. \& Szleifer, I. Optica properties of responsive hybrid Au@Polymer nanoparticles. ACS Nano 6, 8397-8406 (2012)

75. Müller, M. B. et al. Plasmonic library based on substrate-supported gradiential plasmonic arrays. ACS Nano 8, 9410-9421 (2014).

76. Telis, V. R. N., Telis-Romero, J., Mazzotti, H. B. \& Gabas, A. L. Viscosity of aqueous carbohydrate solutions at different temperatures and concentrations. Int. J. Food Prop. 10, 185-195 (2007).

77. Surendar, C. \& Raju, D. Determination of refractive index of glucose solution using low cost fiber optic sensor. Int. J. Eng. Sci. Res. Technol. 5, 155-159 (2016).

78. Martinsson, E., Otte, M. A., Shahjamali, M. M., Sepulveda, B. \& Aili, D. Substrate effect on the refractive index sensitivity of silver nanoparticles. J. Phys. Chem. C. 118, 24680-24687 (2014).

79. Burns, D. T. \& Walker, M. J. Origins of the method of standard additions and of the use of an internal standard in quantitative instrumental chemical analyses. Anal. Bioanal. Chem. 411, 2749-2753 (2019).

80. Sarigul, N., Korkmaz, F. \& Kurultak, I. A new artificial urine protocol to better imitate human urine. Sci. Rep. 9, 20159 (2019).

81. Miyashita, M. et al. Development of urine glucose meter based on micro-planer amperometric biosensor and its clinical application for self-monitoring of urine glucose. Biosens. Bioelectron. 24, 1336-1340 (2009). 